\title{
Stomatin modulates adipocyte differentiation through ERK pathway and regulates lipid droplet growth and function
}

\section{Shao-Chin Wu}

National Yang-Ming University/Biophotonics https://orcid.org/0000-0002-1960-1581

\section{Yuan-Ming Lio}

National Yang-Ming University/Microbiology and Immunology

Jui-Hao Lee

National Yang-Ming University/Taiwan International Graduate Program in Molecular Medicine

Chin-Yau Chen

National Yang-Ming University Hospital

Tung-Wei Chen

National Yang-Ming University

Hong-Wen Liu

National Yang-Ming University

Wei-Nan Lian

National Yang-Ming University

Wei-Ju Lin

National Yang-Ming University/Cancer Progression Research Center

Chih-Yung Yang

Taipei City Hospital

Chien-Yi Tung

National Yang-Ming University/Cancer Progression Research Center

Chi-Hung Lin ( $\square$ linch@ym.edu.tw)

National Yang-Ming University/Biophotonics

\section{Article}

Keywords: Stomatin, adipocyte differentiation, ERK pathway, lipid droplet growth and function

Posted Date: November 10th, 2020

DOI: https://doi.org/10.21203/rs.3.rs-94915/v1 
License: (c) (i) This work is licensed under a Creative Commons Attribution 4.0 International License. Read Full License

Version of Record: A version of this preprint was published at Nature Communications on July 19th, 2022. See the published version at https://doi.org/10.1038/s41467-022-31825-z. 
1 Stomatin modulates adipocyte differentiation through ERK pathway and regulates lipid droplet growth and function

3 Shao-Chin Wu ${ }^{1}$, Yuan-Ming Lio ${ }^{2}$, Jui-Hao Lee ${ }^{3}$, Chin-Yau Chen ${ }^{4}$, Tung-Wei Chen ${ }^{1}$, 4 Hong-Wen Liu ${ }^{5}$, Wei Nan Lian², Wei-Ju Lin ${ }^{6}$ Chih-Yung Yang ${ }^{7}$, Chien-Yi Tung ${ }^{6 \#}$, Chi5 Hung Lin ${ }^{1,2,6,8 *}$

6 1. Institute of Biophotonics, National Yang-Ming University, Taipei, Taiwan

7 2. Institute of Microbiology and Immunology, National Yang-Ming University, Taipei, Taiwan

8 3. Taiwan International Graduate Program in Molecular Medicine, National Yang-Ming University

9 and Academia Sinica, Taipei, Taiwan

10 4. National Yang-Ming University Hospital, Yilan, Taiwan

11 5. Chong Hin Loon Memorial Cancer and Biotherapy Research Center, Taipei, Taiwan

12 6. Cancer Progression Research Center, National Yang-Ming University, Taipei, Taiwan

13 7. Department of Education and Research, Taipei City Hospital, Taipei, Taiwan

14 8. Department of Biological Science and Technology, National Chiao-Tung University, Hsinchu,

15 Taiwan

$16{ }^{*}$ Correspondence

17 Chi-Hung Lin, MD, ph.D.

18 Institute of Biophotonics, National Yang-Ming University, Taipei, Taiwan

19 Institute of Microbiology and Immunology, National Yang-Ming University, Taipei, Taiwan

20 Cancer Progression Research Center, National Yang-Ming University, Taipei, Taiwan

21 Department of Biological Science and Technology, National Chiao-Tung University, Hsinchu,

22 Taiwan

23 No.155, Sec.2, Linong Street, Taipei, 112 Taiwan (ROC)

24 Telephone: +886-2-28267000\#5613

25 E-mail: linch@ym.edu.tw

26 \#Co-correspondence

27 Chien-Yi Tung, ph.D.

28 Cancer Progression Research Center, National Yang-Ming University, Taipei, Taiwan

29 No.155, Sec.2, Linong Street, Taipei, 112 Taiwan (ROC)

30 Telephone: $+886-2-28267000 \# 5678$ 
31 E-mail: E-mail: Cytung@ym.edu.tw 


\section{Abstract}

34 Controlling fatty acid uptake, lipid production and storage, and metabolism of lipid 35 droplets (LDs), are closely related to lipid homeostasis, adipocyte hypertrophy and 36 obesity. We report here that stomatin, a major constituent of the lipid raft, participate 37 in adipogenesis and lipogenesis by preferentially recruiting effectors, such as perilipin 38 for LD fusion or transporters for fatty acid uptake. Adipocyte-like cells having increased 39 stomatin expressions exhibit higher levels of fatty acid uptake and LD growth or 40 enlargements. Moreover, transgenic mice fed with a high-fat diet showed increased stomatin expression that facilitated progression of obesity and caused insulin

42 resistance and hepatic impairments. Conversely, inhibitions of stomatin by gene

43 knockdown or pharmacological treatments could block not only LD growth but also 44 adipogenic differentiation through downregulation of PPARy pathway. Effects of 45 stomatin on PPARY involved ERK signaling; however, an alternate pathway also exist. 46 Amongst various anti-obesity measures, stomatin serves as another potential 47 therapeutic target.

\section{Introduction}

49 Stomatin is an ancient, widely expressed, oligomeric, monotopic integral membrane 50 protein that is associated with cholesterol-rich membrane microdomains/lipid rafts. 51 Besides being a major component of the lipid raft, functions of stomatin are largely 52 unclear. The gene was first identified as the causal factor of Overhydrated Hereditary 53 Stomatocytosis (OHSt) disease, and was therefore named "stomatin" 1, 2. However, 54 stomatin knockout mouse was viable and did not show stomatocytosis ${ }^{3}$. Human stomatin is ubiquitously expressed; high expressions are noted in adipose tissues, 56 bone marrow and placenta (Supplemental Fig. 1). In placenta, stomatin plays an 57 important role in trophoblast differentiation ${ }^{4}$; and in bone, stomatin promotes osteoclastogenesis ${ }^{5}$. At the cell level, stomatin is associated with the plasma 59 membrane and cytoplasmic vesicles, such as endosomes ${ }^{6}$, lipid droplets (LDs) ${ }^{7}$, and 60 specialized endosomes/granules in hematopoietic cells ${ }^{8}$. We have previously 61 reported that stomatin, with its unique molecular topology, promoted cell-cell fusion by 62 forming molecular assembly that recruited fusogenic protein to the appositional 63 plasma membranes ${ }^{5}$. In addition to the regulations of fusion events, stomatin can also 64 interact with various plasma membrane proteins residing within lipid raft and modulate 
65 their activities. For examples, stomatin can regulate the transport activities of Anion 66 Exchanger $1(\mathrm{AE} 1)^{9}$, glucose transporter (GLUT1) ${ }^{10}$ and water channel aquaporin-1

67 (AQP1) ${ }^{11}$, or activity of acid-sensing ion channel (ASIC) family ${ }^{12,13,14}$. Whether such regulations are mediated by stomatin's scaffolding effects that change the biophysical

69 properties of the lipid rafts or by direct protein-protein interactions between stomatin 70 and the effector molecules, are currently unknown. It is also unclear if and how 71 stomatin is involved in signal transduction which is initiated by complex protein-protein 72 interactions between ligands, receptors and signaling molecules that may occur in the vicinity of lipid rafts. It has been speculated that the size and composition of lipid rafts could change in response to intra- or extracellular stimuli; such changes might favor specific protein-protein interactions and activate corresponding signaling cascades. In this study, we addressed the roles of stomatin in adipocyte differentiation and functions, focusing on intracellular vesicular fusion among LDs, fatty acid uptake and their associated signal transduction.

80 Obesity, resulting from a positive energy balance between energy harvest and energy expenditure ${ }^{15,16}$, is a prevalent global healthcare problem that continues to increase in countries around the world ${ }^{17}$. Obesity is characterized by increased adipose tissue mass that has been associated with a strong predisposition towards metabolic diseases such as diabetes, cardiovascular diseases, non-alcoholic fatty liver diseases and some cancers ${ }^{18,19,20}$. The expansion of adipose depots, especially the white adipose tissues, are characterized either by the increase in adipocyte size (hypertrophy) or by the formation of new adipocytes from precursor differentiation in the process of adipogenesis (hyperplasia). Genetic and behavioral attributors both contribute to obesity-related energy imbalance ${ }^{21,22}$. In the presence of excessive energy, mature adipocytes increase in cell size and undergo cellular hypertrophy to store the surplus fat ${ }^{23}$.

93 Hypertrophic adipose morphology is positively associated with insulin resistance, 94 diabetes and cardiovascular disease. The hypertrophic adipocytes are ultimately 95 responsible for dysfunction of lipid homeostasis, along with other pathological 96 consequences ${ }^{24}$. Hypertrophic adipocytes are characterized by excessive growth of

97 LDs; the resulting unilocular LD may occupy more than $90 \%$ of the cell volume ${ }^{25}$. The 
98 growth of LDs is achieved by incorporation of triacylglyceride (TG) that is synthesized

99 either locally on the surface of LDs ${ }^{26}$, or obtained from the endoplasmic reticulum ${ }^{27}$.

100 Since fatty acid (FA) is the primary energy source assimilated by adipocytes during 101 lipogenesis, measurements of FA uptake are indicative of LD growth ${ }^{28,29}$. LDs can 102 also grow via fusion of small LDs ${ }^{30}$. Previous studies have shown that several surface 103 proteins of LDs were involved in controlling the LD size in adipocytes. For examples, 104 Perilipin (Plin1), a member of PAT family proteins highly expressed in adipocytes, 105 could regulate the lipolysis functions ${ }^{31,32}$. The cell death-inducing DNA fragmentation 106 factor alpha (DFFA)-like effector (CIDE) family proteins, Cidea, and Fsp27/Cidec, also 107 functioned as LDs size regulators ${ }^{33}, 34$. Cooperative interactions of Plin1 and Fsp27 108 enhanced lipid transfer and LD growth ${ }^{35}$.

110 Adipogenesis is the process by which adipose tissues expand through hyperplastic 111 growth and differentiation. The formation of new adipocytes from precursor cells 112 enhance the capacity of energy storage. Adipogenesis is characterized by 113 expressions of C/EBP $\beta$ and C/EBPD, followed by C/EBPa and PPARy. The master 114 transcription regulator PPARY controls a variety of genes involved in adipogenesis ${ }^{36}$, $115{ }^{37}$. Previous studies have shown that activation of MAPK/ERK pathway (also known 116 as the Ras-Raf-MEK-ERK pathway) could down-regulate transcriptional activity of 117 PPARY ${ }^{38,39}$ and inhibit adipocyte differentiation.

119 Approaches aimed at increasing adipogenesis over adipocyte hypertrophy are now 120 regarded as a means to treat metabolic diseases. Notably, adipocyte expansion 121 through adipogenesis could mitigate the negative metabolic effects of obesity, 122 although the mechanisms and regulators are not fully understood ${ }^{40}$. This study 123 addressed cell biology and signaling pathways of adipocyte-like cells affected by 124 stomatin and provide insight into the control of adipogenesis and hypertrophic 125 adipocytes related to obesity. 


\section{Results}

128 Expressions of Stomatin increased during adipogenic differentiation

129 A cell model for adipogenic differentiation was established ${ }^{41}$. Treating murine 3T3-L1

130 fibroblasts with MDI cocktail for three days (day0 day3), followed by insulin treatment 131 (day3 day7), rendered the cells to differentiate into adipocytes-like cells, as 132 evidenced by lipid accumulation visualized and quantified by Oil Red O staining (Fig.

133 1A). Western blotting assays demonstrated that the expression of stomatin, as well as 134 major adipogenic proteins, such as PPARY, C/EBPa, and Perilipin, progressively 135 increased during adipogenesis (Fig. 1B). After 7-day differentiation, mature adipocytes 136 containing large lipid droplets (LD) were noted under DIC microscopy and stained 137 positive with Oil Red $\mathrm{O}$. Immunofluorescence staining revealed subcellular 138 distributions of stomatin mainly on the vesicular membranes of LDs, colocalized with 139 perilipin proteins (Fig. 1C).

\section{High expressions of Stomatin enhanced LD growth}

141 To understand the roles of stomatin in adipogenic differentiation, we over-expressed 142 human stomatin gene (hSTOM) in murine 3T3-L1 cells. After chemically induced 143 differentiation, we found that the levels of lipid accumulation and adipogenic proteins 144 were very similar in cells having excessive exogenous stomatin proteins, compared to 145 the control (Fig. 2A-B). However, when measuring the size of individual LDs, we 146 noticed that there were more large-sized LDs in cells expressing hSTOM than the 147 control (Fig. 2C), indicating that stomatin were involved in LD growth, or large LD 148 formation. Such dynamics of LDs could result from either fusion of small LDs into a 149 large one (exemplified by Supplemental Video 1 that captures the sequence of a LD150 LD fusion event), or from exchanges of lipid content between interacting LDs.

152 Since small LD vesicles were not discernible under light microscopy, we employed 153 fluorescence recovery after photobleaching (FRAP) experiments to investigate LD 154 activities. As shown in Fig. 2E, photobleaching the fluorescent lipid content of a LD 155 resulted in a fast recovery of fluorescence, reaching half of the original intensity in 15 156 min in cells over-expressing stomatin compared to a very slow recovery, reaching less 
157 than $8 \%$ of original intensity in $15 \mathrm{~min}$, in the control cells. These results indicate an

158 active role of stomatin in facilitating dynamic interactions between LDs.

159 Highly abundant Stomatin facilitates fatty acid uptake both in vitro and in vivo

160 The process of lipid biogenesis, or lipogenesis, starts with either de novo synthesis, 161 or fatty acid uptake from extracellular environment using various fatty acid transporters $162{ }^{42}$. In the experimental assay shown in Fig. $2 F$, fluorescently labeled palmitic acid 163 analogs, BODIPY-C16, when added to the culture medium, were internalized into the 164 cells and quantified continuously using an ELISA reader. Cells having high abundance 165 of stomatin exhibited more and faster fatty acid uptake compared to the control. 166 Moreover, in vivo fatty acid uptake experiments were performed in STOM transgenic 167 (STOM Tg) mice. BSA-emulsified BODIPY-C16 were injected into tail vein; after 15 $168 \mathrm{~min}$, there were much more fluorescence signals found in the adipose tissues of STOM 169 Tg than the control mice (Fig. 3F).

170 High expressions of Stomatin caused obesity in mice fed with high-fat diet

171 We generated STOM transgenic (STOM Tg) mice by engineering human stomatin 172 gene into the animal. These mice contained high amount of hSTOM proteins in 173 subcutaneous white adipose tissues, distributed mainly on the surfaces of white 174 adipocytes (Fig. 3A-B). STOM Tg and the control wild-type (WT) mice were fed either 175 regular chow diet (CD), or high-fat diet (HFD) beginning at 3 weeks of age; the mice 176 were weighed every week. We noticed that body weight gains were about the same 177 comparing STOM Tg with WT mice fed with CD. When fed with HFD, however, mice 178 having up-regulated STOM gene gained more weights more rapidly than their WT 179 littermates (Fig. 3C-D). After 20 weeks, body weights of HFD-fed STOM Tg were at 180 least $20 \%$ higher than WT. Whole-body composition measurements showed that the 181 increase of fat was more significant than increases of lean, free fluid or total water (Fig. 182 3E).

183 HFD-fed STOM Tg mice exhibited adipocyte hypertrophy, impaired metabolisms and hepatic dysfunctions

185 After 20-week HFD feeding, mice having up-regulated STOM gene showed significant 186 increase in subcutaneous adipose tissue (SAT) and brown adipose tissue (BAT) than 
187 WT, whereas mass of visceral adipose tissue (VAT) were about the same (Fig. 4A).

188 Adipocytes from SAT of HFD-fed STOM Tg mice appeared hypertrophic; they were

189 larger in size than those of WT littermates by histogram analyses (Fig. 4B). In addition,

190 Western blotting of 3T3-L1 adipocyte-like cells showed that enzymes for lipolysis,

191 including perilipin and hormone-sensitive lipase (HSL) and its various serine-

192 phosphorylated forms, were similar in cells having over-expressed hSTOM and control

193 cells (Supplemental Fig. 2A), indicating that the observed adipocyte hypertrophy

194 caused by stomatin was not due to impaired triglyceride metabolism.

195

196 The observed obesity was not a result of altered energy expenditure or energy

197 substrate selection, as evidenced by similar respiratory exchange rates comparing

198 HFD-fed STOM Tg mice and their littermate controls (Supplemental Fig. 2B). The

199 calculated heat production during the light-dark cycle showed that HFD-fed STOM Tg

200 mice had only slightly higher heat production which was too little to influence obesity

201 progression (Supplemental Fig. 2C).

203 Biochemical analysis after 20-week HFD feeding showed that total cholesterol (TCHO)

204 of HFD-fed STOM Tg were slightly higher than the control, but triglycerides (TG) levels 205 were the same (Supplemental Fig. 2D). Interestingly, although fasting blood glucose 206 were similar between the groups, plasma insulin and insulin resistance measured by 207 HOMA-IR experiments, and glucose tolerance measured by intraperitoneal glucose 208 tolerance test (IPTGG) were much higher and intolerable in STOM Tg than WT mice 209 (Fig. 4C). These results indicated that HFD-fed, STOM Tg aggravated glucose 210 homeostasis by enhancing insulin resistance.

212 Obesity is usually correlated with ectopic fat accumulation in the liver. Indeed, HFD-

213 fed STOM Tg possessed not only larger liver mass (hepatomegaly), but also exhibited 214 phenotypes of macro- and microvesicular steatosis. The observed hepatic steatosis 215 appeared to affect liver functions, as evidenced by elevated levels of plasma GPT and 216 GOP in HFD-fed STOM Tg mice (Fig. 4D). 


\section{Knockdown of STOM inhibited adipogenesis and LD growth}

218 Knockdown of STOM expression was done by short hairpin RNA (shRNA) method.

219 Two shRNAs designed to target different sites of murine stomatin were separately 220 packaged into lentiviral particles and introduced into 3T3-L1cells, resulting in 221 shSTOM-1 and shSTOM-2 cells. Both shRNAs could effectively down-regulate 222 stomatin expressions. Knocking-down STOM expression inhibited not only 223 adipogenesis, evidenced by lack of lipid accumulation (Fig. 5A) and inhibitions of 224 genes involved in adipocytic differentiation, such as PPARY and C/EBPa (Fig. 5B). 225 Knockdown of STOM also inhibited LD growth. As shown by Fig. 5C, histogram 226 analyses of LD sizes revealed more small-LD and fewer large-LD following STOM 227 knockdown.

229 Differential expressions of shSTOM-1 and control cells were analyzed after induction 230 of adipogenic differentiation for seven days by the microarray method. A total of 1478 231 annotated coding genes were identified from the transcriptomes by a stringent 232 threshold of $P$ value $<0.05$ and FDR $P$ value $<0.001$. Among them, 379 transcripts 233 were of significant difference between Stom-deficient and control cells (fold change $\geq$ 2343 , or $\leq-3$ ); 185 of them were up-regulated and 194 down-regulated by inhibition of 235 stomatin expression (Supplemental Fig. 3A). The global view of these genes was 236 constructed by hierarchical clustering to characterize changes across six samples 237 (Supplemental Fig. 3B). These genes were mapped onto 128 Wikipathways using 238 Transcriptome Analysis Console (TAC). As the top-tanking enriched pathways 239 shown (Fig. 5D), adipogenesis genes were the most profoundly inhibited gene groups, 240 followed by PPAR signaling pathway. In the "adipogenesis genes" pathway, 12 genes 241 involved in this pathway showed significant changes; 9 of them $(75 \%)$ were down242 regulated and $3(25 \%)$ were up-regulated (Fig. 5E). To further validate the results, we 243 performed qPCR experiments for Pparg and Cebpa genes and confirmed 244 transcriptomic findings (Fig. 5F).

245 Somatin inhibitor OB-1 affected adipogenic differentiation and inhibited LD 246 growth

247 By inhibiting self-association, OB-1 is an effective inhibitor of stomatin-mediated 248 functions ${ }^{43}$. LD 50 for OB-1 in 3T3-L1 cells was first determined (Fig. 6A). Drug 
249 treatments using $25 \mu \mathrm{M}$ OB-1, while maintaining good cell viability, inhibited 250 adipogenic differentiation in a dose-dependent manner as evidenced by decreased 251 lipid accumulation (Fig. 6B). Treating 3T3-L1 cells during adipocytic differentiation with 252 OB-1 also inhibited LD growth; histogram analyses of LD sizes were applied to 253 quantify the increase of small LDs and decrease of large LDs (Fig. 6C).

\section{Stomatin modulated adipocyte differentiation through ERK pathway}

255 Inhibitions of stomatin by either shRNA (Fig. 5A) or OB-1 (Fig. 6B) could effectively 256 block lipid accumulation by adipocytic 3T3-L1 cells. Using Western blotting assays, 257 we analyzed their effects on other adipogenesis-related genes and signaling pathways 258 (Fig. 7). Both stomatin-deficient shSTOM-1 and shSTOM-2 cells exhibited decreased 259 PPARY protein. While the Akt pathway was not affected by STOM knockdown, the 260 ERK pathway appeared activated as evidenced by the increased level of 261 phosphorylated ERK (arrow, Fig. 7A). OB-1 treatment also caused increase of 262 phospho-ERK (Fig. 7D).

264 Two early adipogenesis genes, C/EBP $\beta$ and C/EBPD, are upstream of PPARY 265 regulation. After induction of adipogenic differentiation, C/EBP $\beta$ and C/EBPס, 266 exhibited a transient increase in the first three days, followed by a gradual decrease 267 from day 3 to day 7 (Fig. 7B and 7C, respectively). Knockdown of stomatin had little 268 effect on the protein levels and degrees of C/EBP $\beta$ phosphorylation (data not shown); 269 in contrast, inhibition of stomatin was able to maintain C/EBPס expression at much 270 higher level than the control (Fig. 7B-C).

272 Since activation of ERK pathway has been shown to inhibit PPARY ${ }^{44}$, we examined 273 whether stomatin positively regulated PPARy regulation via inhibition of pERK (red 274 arrows, Fig. 8). To this end, we treated shSTOM-1 cells with U0126, a highly selective 275 inhibitor for ERK, under the notion that U0126 might mitigate the stomatin-knockdown 276 effect of pERK activation. However, we found that U0126 treatment could not reverse 277 the lipogenesis-deficit phenotype of hSTOM-1 cells (Fig. 7E). In contrast, treating 278 hSTOM-1 cells with troglitazone (TGZ), a PPARy agonist, was able to partially rescue 279 lipogenesis-deficit of hSTOM-1 cells; and interestingly, dual treatments of U0126 and 280 TGZ were noted to further recover lipid accumulation (Fig. 7F). These results suggest 
281 the presence of a currently unknown mechanism for stomatin to positively regulate 282 PPARy and activate adipogenesis (black arrows, Fig. 8); this unknown pathway may work synergistically with the ERK pathway.

\section{Discussion}

285 We propose a working model for the roles of stomatin in adipogenic differentiation and 286 lipogenesis (Fig. 8). Along the differentiation process from undifferentiated progenitor 287 cells to immature adipocytes then to mature adipocyte, expression of stomatin 288 progressively increase. C/EBP $\beta$ and C/EBP $\delta$ exhibits a transient increase during the 289 early phase, while C/EBPa in the later phase. The various adipogenic genes and 290 signaling processes seem to converge onto the PPARy pathway, which is considered 291 one of the master regulators of adipocyte differentiation ${ }^{37}$. We report a novel finding 292 that knockdown of stomatin was able to activate ERK signaling as evidenced by the 293 increase of pERK. Since pERK is a well-known negative regulator of PPARY we 294 wonder if the promoting effect of stomatin on PPARY and the downstream adipogenic 295 phenotype is mediated through downregulation of pERK (red arrows, Fig. 8, see also

$296{ }^{44}$ ). Our results (Fig. 7E-F), however, did not support this notion. Unlike activation of 297 PPARY, activation of ERK pathway alone did not rescue the lipogenesis deficit caused 298 by stomatin knockdown. This suggests the presence of another currently unknown 299 pathway (black arrow, Fig. 8) that may work synergistically with ERK pathway to 300 activate PPARY and by doing so promote adipogenesis and lipogenesis.

302 What signaling events are affected by stomatin which serves a major role as a 303 component of lipid rafts? Through transcriptome and pathway analyses, we identified 304 MAPK signaling as a potential pathway affected by stomatin (Fig. 5D-E and supplemental Fig. 3). In the case of adipocyte differentiation, EGFR is one of the upstream receptor tyrosine kinases (RTKs) for MAPK signaling. RTKs control many 307 fundamental cell behaviors by activating a series of downstream signaling pathways 308 including RAS-RAF-MEK-ERK pathway or AKT-PI3K-mTOR pathway ${ }^{45,46}$. We found 309 that the RAS-RAF-MEK-ERK pathway is involved in stomatin-mediated adipogenic 310 differentiation (Fig. 7A). Also, EGFR metabolism is highly regulated by lipid-raft 311 mediated internalization, recycling and degradation ${ }^{47,48,49,50}$. Increased expression of 312 stomatin, for example, caused a reduction in EGFR on the plasma membrane 
313 (unpublished data), resulting in reduced ERK activity which is necessary for adipocyte

314 differentiation ${ }^{45}$.

316 Besides participating in modulation of signaling during adipogenic differentiation, 317 stomatin also plays a crucial role in LD growth characterized by vesicle enlargement 318 and transformation from multilocular LDs to unilocular LDs typically seen in adipose 319 tissues. LD is a universal cellular organelle that responds to lipid storage. It is believed

320 to be generated from the endoplasmic reticulum (ER) ${ }^{51}$ and can enlarge through the 321 incorporation of lipid from the ER ${ }^{52}$, the local synthesis of triacylglycerols (TAGs) ${ }^{26}$, $322{ }^{53}$, and the fusion of multiple lipid droplets ${ }^{54}$. Stomatin, as a LD-associated protein ${ }^{7}$ 323 and a major lipid raft component, could play multiple roles in growth of LD. First, 324 stomatin if present in high abundance could promote formation of potential fusion 325 pores that leads to LD fusion ${ }^{4,5}$. Second, stomatin could recruit fusion facilitators to 326 LD-LD contact site (LDCS), and by doing so regulate LD-fusion and growth. Third, 327 stomatin could help generate passage tunnels that allow lipid exchange among 328 contacted LDs ${ }^{35,55}$. Due either to illumination toxicity to the cell or the fact that the 329 majority LD undergoing fusion were of submicron-sized, direct imaging of fusion 330 between micron-sized LD was hard to achieve under light microscopy. We therefore 331 employed FRAP experiments to address the third mechanism. Lipids in the smaller 332 LDs of the contacted pair are transferred to the larger LDs, owing to the internal 333 pressure difference, thus resulting in the fusion and growth of LDs.

335 For the aforementioned second mechanism, we have previously reported that 336 stomatin works as an "enhancer" on the plasma membrane to increase the 337 effectiveness of molecular machinery for membranes fusion ${ }^{4,5}$. Within a lipid raft, for 338 example, stomatin can restrict existing fusogenic effectors to the sites of membrane339 membrane contact and enhance their interactions. The same mechanism may apply 340 to intracellular inter-vesicular fusions. Locally enriched stomatin and perilipin can 341 recruit cell death-inducing DFF45-like effector (CIDE) family proteins to LDCS ${ }^{35}$. As 342 CIDE family proteins are crucial regulators of LD-fusion ${ }^{56}$, the "fencing" mechanism 343 by stomatin can promote fusion pore expansion at LDCS, resulting in LD fusion and 344 growth. 
346 The ability of adipocyte to uptake fatty acid of superfluous lipids from the extracellular

347 environment is impacted by their physiological functions in energy homeostasis. The

348 amount and rate of fatty acid uptake were noted to increase in the presence of high

349 level stomatin both in vitro and in vivo. Although some fatty acids can cross plasma

350 membrane by passive diffusion ${ }^{57}$, most fatty acid uptake is mediated by membrane-

351 associated transporters; many of them reside and function in the lipid rafts. When

352 bound to long-chain fatty acid (LCFAs), FAT/CD36 may partition into lipid rafts to

353 accelerate the translocation of LCFAs ${ }^{28,58}$. In lipid rafts, stomatin can function as an

354 anchor or organizer for these cholesterol-rich membrane domains. Stomatin can also

355 modulate the function of effector residing within lipid rafts. For example, stomatin can

356 regulate several ion channels activities ${ }^{59,60}$ and glucose transport GLUT1 functions ${ }^{10}$.

357 In a more general sense, stomatin can capture or trap the lateral diffusion of proteins

358 within the lipid raft or affect the interaction between ligand and receptors, and by doing

359 so, regulate the downstream signaling transduction.

Adipose tissues play a central role in regulating energy storage to protect other tissues, such as the muscle and the liver, from the harmful effects of superfluous circulating free fatty acid. So, fatty acid uptake and LD growth are crucial in controlling lipid storage and obesity development. We demonstrated here that high levels of stomatin, combined with chronic energy surplus, may lead to a hypertrophy phenotype of adipocytes (the HFD-fed STOM Tg mice). The resulting limitation in fat storage capacity then cause local and systemic metabolism disorders ${ }^{61}$. In a broader sense, stomatin is involved in regulating various aspects of lipid homeostasis, including adipocyte differentiation, lipid production, lipid storage, lipolysis and lipid secretion.

370 The finding that stomatin regulates fatty acid uptake and LD growth may provide new

371 opportunities for correcting whole-body energy disorders or energy surplus-induced

372 obesity by modulating the molecular events associated with stomatin. 


\section{Methods}

\section{Cell Culture and induction of adipogenic differentiation}

376 3T3-LI murine fibroblasts, purchased from Bioresource Collection and Research 377 Center (no.60159; BCRC, Hsinchu, Taiwan), were maintained in high-glucose 378 Dulbecco's modified Eagle's medium (DMEM) supplemented with $10 \%$ bovine 379 calf serum and $1 \%$ sodium pyruvate. To induce differentiation into adipocyte-like cells, 380 3T3-LI cells were first grown to confluence. Two days after reaching confluence, the 381 medium was replaced with DMEM supplemented with $10 \%$ fetal bovine serum (FBS) 382 containing MDI cocktail, including $0.5 \mathrm{mM}$ IBMX, $0.25 \mu \mathrm{M}$ dexamethasone and 10 $383 \mu \mathrm{g} / \mathrm{ml}$ bovine insulin. After 72 hours, the medium was replaced with DMEM 384 supplemented with $10 \%$ FBS and $10 \mu \mathrm{g} / \mathrm{ml}$ bovine insulin. The medium was refreshed 385 every other day. Human embryonic kidney (HEK) 293T cells were cultured in DMEM 386 supplemented with $10 \%$ FBS.

\section{Lentivirus production and transduction}

388 Lenti-Vector pLAS2W.puro, shRNA for Stom vectors (shSTOM-1: clone number 389 TRCN0000112911 and shSTOM-2: clone number TRCN 0000112912) and packaging 390 plasmids pCMV delta R8.91 and PMD.G were obtained from National RNAi 391 Core Facility Platform (Acadamic Sinica, Taiwan). RFP, hSTOM-RFP and hSTOM392 flag (hSF) were amplified from expression plasmids and cloned into the lenti-Vector. 393 HEK-293T cells were co-transfected with lentivector and packaging plasmids using 394 NTR II Non-liposome transfection reagent II (T-Pro-Biotechnology, Taiwan). The 395 supernatants containing lentivirus were harvested after 24 - and 48-hr and centrifuged 396 at $1,250 \mathrm{rpm}$ for $5 \mathrm{~min}$ to exclude any remaining HEK-293T packaging cells. 397 Subsequently, cells were transduced with the lentivirus-containing supernatants for 24 $398 \mathrm{hrs}$, followed by $3 \mu \mathrm{g} / \mathrm{mL}$ puromycin selection for 3 days.

\section{Quantifications of lipid accumulation}

400 Oil Red O stock solution was prepared by dissolving 0.35g Oil Red O (no. O0625, 401 Sigma Aldrich) in $100 \mathrm{ml}$ pure isopropanol. Cells were fixed with $3.7 \%$ formaldehyde 402 for $1 \mathrm{hr}$ at room temperature, followed by two times wash, then dried entirely using a 403 hairdryer. Post-fixed cells were stained with Oil Red O, diluted in distilled water (6:4) 
404 for $1 \mathrm{hr}$ at room temperature, further washed four times with distilled water. The stained

405 dyes in lipid droplets were extracted with pure isopropanol and absorbance at $490 \mathrm{~nm}$ 406 (OD 490) was measured.

\section{Western blot analysis}

408 Cultured cells were washed twice with cold PBS, then lysed using RIPA Iysis buffer 409 supplemented with cOmplete ${ }^{\mathrm{TM}}$ (EDTA-free Protease Inhibitor Cocktail) and 410 PhosSTOP тM (both from Sigma Aldrich). The amount of total extracted proteins was 411 quantified using Pierce $660 \mathrm{~nm}$ protein assay reagent (Thermo Fisher Scientific ${ }^{\mathrm{TM}}$ ). 412 The protein lysates were separated by SDS-PAGE, then transferred onto PVDF 413 membranes. The membranes were blocked with $5 \%$ bovine serum albumin (BSA) in 414 TBS-T (Tris-buffer saline supplement with $0.1 \%$ Tween 20) at room temperature for 1 $415 \mathrm{hr}$, and then incubated with primary antibodies, such as anti- STOM (a166623b, 416 Abcam), anti-perilipin (9349, Cell Signaling), anti- PPARY (2435, Cell Signaling), anti417 C/EBPa (8178, Cell Signaling), anti-human STOM (M-14, Santa Cruz Biotechnology),

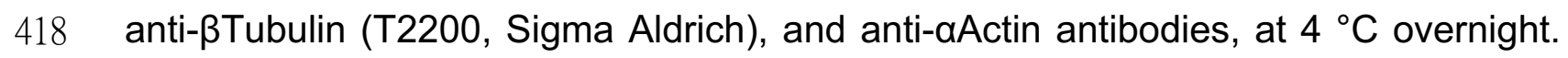
419 After washing three times with TBS-T, the PVDF membranes were incubated with 420 horseradish peroxidase-conjugated secondary antibodies for $1 \mathrm{hr}$ at room temperature, 421 then treated with substrates (SuperSignal West Femto Maximum Sensitivity Substrate, 422 Thermo Fisher Scientific), then visualized by ImageQuant (GE Healthcare Life 423 Science).

\section{Immunofluorescence}

425 Cells seeded on coverslips were fixed with 3.7\% paraformaldehyde (Sigma-Aldrich) at 426 room temperature for $15 \mathrm{~min}$, rinsed twice with PBS for $5 \mathrm{~min}$, permeabilized and 427 blocked using extraction buffer composed of $0.1 \%$ saponin (Sigma-Aldrich) and $1 \%$ 428 BSA in PBS, for $1 \mathrm{hr}$. Cells were then incubated with primary antibodies, including anti429 STOM (a166623b, Abcam) and anti-perilipin (GT2781,GeneTex), at $4{ }^{\circ} \mathrm{C}$ overnight. 430 After washing three times with wash buffer (PBS with $0.1 \%$ saponin), cells were 431 incubated with fluorochrome-conjugated secondary antibodies for $1 \mathrm{hr}$, and observed 432 under a confocal microscope (LSM700, Zeiss). 


\section{FRAP assay}

434 Adipocyte-like 3T3-L1 cells were incubated with $6 \mu \mathrm{M}$ of BODIPY ${ }^{\mathrm{TM}}-\mathrm{FL}_{-} \mathrm{C}_{12}$ (D3822, 435 Invitrogen ${ }^{\mathrm{TM}}$ ) at $37^{\circ} \mathrm{C}$ overnight. After refreshing with complete medium, live cells were 436 viewed under a confocal microscope (Zeiss, LSM700) using a 100x oil immersion 437 objective. Selected regions were first bleached with 15 pulses of $100 \%$ laser power 438 (combined 488 with 405 diode lasers), followed by time-lapse recording at 30-sec 439 interval using normal imaging laser power.

\section{Fatty acid uptake assay}

441 For in vitro analysis, adipocyte-like 3T3-L1 cells were serum-starved in serum-free 442 DMEM containing $1 \%$ sodium pyruvate for $1 \mathrm{hr}$ at $37^{\circ} \mathrm{C} / 5 \% \mathrm{CO} 2$, changed to serum443 free DMEM containing $1 \%$ sodium pyruvate and $10 \mu \mathrm{g} / \mathrm{ml}$ insulin for $30 \mathrm{~min}$, then to 444 1X HBSS containing 20mM HEPES. Subsequently, 0.2 $\mu$ M BODIPY-FL-C 16 (Thermo) 445 was added. The fluorescence (excitation: $485 \mathrm{~nm}$ and emission: $515 \mathrm{~nm}$ ) was detected 446 by ELISA reader (TECAN) using bottom-read mode and kinetic reading at a 30-sec 447 interval. For in vivo analysis, $400 \mathrm{nM}$ BODIPY ${ }^{\mathrm{TM}}-\mathrm{FL}_{-}-\mathrm{C}_{16}$ was emulsified in $65 \mathrm{mg} / \mathrm{ml}$ 448 BSA solution to prepare the probe solution. An amount of $1.2 \sim 1.5 \mathrm{ml}$ (6 8\% of body 449 weight) probe solution was injected into the animal via tail vein. The mice were 450 sacrificed and subcutaneous adipose tissues were resected for quantifications of 451 fluorescence. Fat pads were homogenized using homogenizer (Roche MagNA Lyser 452 Benchtop Homogenizer) for 45-sec at 3,000 r.p.m., and incubated at $65^{\circ} \mathrm{C}$ for $30 \mathrm{~min}$. 453 After centrifugation at 12,000rpm for $10 \mathrm{~min}$, fat layer was collected and subjected to 454 fluorescence signal quantifications.

\section{STOM transgenic mice and phenotype analyses}

456 Transgenic mice overexpressing human STOM were generated by pronuclear 457 microinjection. STOM was cloned into vector STOM-p1033, and RNA polymerase II 458 large subunit promoter was used to drive expression in C57BL/6 mice as described 459 previously ${ }^{5}$. The integration of the transgene was confirmed by PCR analysis of 460 mouse tail DNA. Three to four weeks male STOM Tg and wild type littermates were 461 fed with either chow diet or high-fat diet (D12492, $60 \mathrm{kcal} \%$ fat, Research Diets) for 462 up to 20 weeks under free-feeding conditions. All of the mice were housed on a 12-hr 463 light/dark cycle at $22^{\circ} \mathrm{C}$. The blood biochemistry of STOM Tg and WT mice were 
464 examined: fasting blood glucose and plasma insulin was determined by human blood 465 glucose meter (Accu-chek Performa, Roche) and Mouse Ultrasensitive Insulin ELISA 466 (80-INSMSU-E01, ALPCO), respectively. GOT and GPT were measured using 467 Automated Clinical Chemistry Analyzer (FUJI DRI-CHEM 4000i).

\section{Glucose tolerance tests}

469 Mice fasted overnight were injected intraperitoneally with glucose solution (1 $\mathrm{g} / \mathrm{kg}$ body 470 weight). Blood glucose levels were measured at 0, 15, 30, 60, and $120 \mathrm{~min}$ after 471 injection using a glucometer.

\section{Histological analyses}

473 The liver and adipose tissues were formalin-fixed and paraffin-embedded. Sections of 474 WAT and the liver were stained with hematoxylin and eosin (H\&E stain). All tissue 475 images were obtained using the Aperio CS2 Digital Pathology Scanner (Leica) and 476 analyzed via Imagescope software. The size of adipose cells was analyzed using the 477 Fiji Adiposoft software to obtain the area of each and individual adipocyte.

\section{Transcriptome expression analyses}

479 Total RNA were extracted from cells using TRIzol® reagent and subjected to global 480 transcriptome analysis using Mouse Genome Arrays (MTA-1_0, Affymetrix). The 481 Transcriptome Analysis Console (TAC4.0) was used to process and analyze CEL files. 482 We initially filtered for 11,264 probe sets annotated as locus type "coding". 483 Subsequently, 1,478 annotated coding genes were identified via exclusively a 484 stringent threshold of $p$-value $<0.05$ and FDR $p$-value $<0.001$. Additionally, we further 485 filtered for 379 genes via exclusively a stringent fold change threshold $\geq 3$, or $\leq-3$. 486 The results were confirmed using qRT-PCR. The cDNAs were generated using 487 SuperScript III First-Strand Synthesis System following the manufacturer's protocol. 488 To determine the expression of specific genes, quantitative real-time PCR (qRT-PCR) 489 was performed using diluted cDNA in a total volume of 5ul with SYBR Green (Qiagen). 490 Gene expression was normalized to the internal reference gene Nono, followed by 491 calculation using $\Delta \Delta \mathrm{CT}$ method. 


\section{References}

495 1. Gallagher PG, Forget BG. Structure, organization, and expression of the human band 7.2b 496 gene, a candidate gene for hereditary hydrocytosis. The Journal of biological chemistry 270 , $497 \quad 26358-26363(1995)$.

498

499 2. Fricke B, et al. The "stomatin" gene and protein in overhydrated hereditary stomatocytosis. Blood 102, 2268-2277 (2003).

501

3. Zhu Y, et al. Stomatocytosis is absent in "stomatin"-deficient murine red blood cells. Blood 93, 2404-2410 (1999).

4. Chen TW, Liu HW, Liou YJ, Lee JH, Lin CH. Over-expression of stomatin causes syncytium formation in nonfusogenic JEG-3 choriocarcinoma placental cells. Cell biology

5. Lee JH, et al. Lipid raft-associated stomatin enhances cell fusion. FASEB journal : official publication of the Federation of American Societies for Experimental Biology 31, 47-59 international 40, 926-933 (2016). (2017).

7. Umlauf E, Csaszar E, Moertelmaier M, Schuetz GJ, Parton RG, Prohaska R. Association of stomatin with lipid bodies. The Journal of biological chemistry 279, 23699-23709 (2004).

8. Mairhofer M, Steiner M, Mosgoeller W, Prohaska R, Salzer U. Stomatin is a major lipidraft component of platelet alpha granules. Blood 100, 897-904 (2002).

523 9. Genetet S, Desrames A, Chouali Y, Ripoche P, Lopez C, Mouro-Chanteloup I. Stomatin modulates the activity of the Anion Exchanger 1 (AE1, SLC4A1). Scientific reports 7, 46170 (2017). 
527 10. Montel-Hagen A, et al. Erythrocyte Glut1 triggers dehydroascorbic acid uptake in mammals unable to synthesize vitamin C. Cell 132, 1039-1048 (2008).

530 11. Rungaldier S, Oberwagner W, Salzer U, Csaszar E, Prohaska R. Stomatin interacts with GLUT1/SLC2A1, band 3/SLC4A1, and aquaporin-1 in human erythrocyte membrane domains. Biochimica et biophysica acta 1828, 956-966 (2013).

13. Klipp RC, Cullinan MM, Bankston JR. Insights into the molecular mechanisms underlying

14. Moshourab RA, Wetzel C, Martinez-Salgado C, Lewin GR. Stomatin-domain protein interactions with acid-sensing ion channels modulate nociceptor mechanosensitivity. The Journal of physiology 591, 5555-5574 (2013).

15. Schwartz MW, et al. Obesity Pathogenesis: An Endocrine Society Scientific Statement. Endocr Rev 38, 267-296 (2017).

19. Wabitsch M, et al. Severe Early-Onset Obesity Due to Bioinactive Leptin Caused by a p.N103K Mutation in the Leptin Gene. J Clin Endocrinol Metab 100, 3227-3230 (2015). 
560 21. Albuquerque D, Stice E, Rodriguez-Lopez R, Manco L, Nobrega C. Current review of genetics of human obesity: from molecular mechanisms to an evolutionary perspective. $\mathrm{Mol}$

22. Xia Q, Grant SF. The genetics of human obesity. Ann N Y Acad Sci 1281, 178-190 (2013).

23. McLaughlin T, et al. Adipose Cell Size and Regional Fat Deposition as Predictors of Metabolic Response to Overfeeding in Insulin-Resistant and Insulin-Sensitive Humans. Diabetes 65, 1245-1254 (2016).

25. Pilch PF, et al. Cellular spelunking: exploring adipocyte caveolae. J Lipid Res 48, 2103-

26. Kuerschner L, Moessinger C, Thiele C. Imaging of lipid biosynthesis: how a neutral lipid enters lipid droplets. Traffic 9, 338-352 (2008). 2111 (2007).

24. Haczeyni F, Bell-Anderson KS, Farrell GC. Causes and mechanisms of adipocyte enlargement and adipose expansion. Obes Rev 19, 406-420 (2018).

28. Ehehalt R, Fullekrug J, Pohl J, Ring A, Herrmann T, Stremmel W. Translocation of long

27. Yang H, Galea A, Sytnyk V, Crossley M. Controlling the size of lipid droplets: lipid and protein factors. Curr Opin Cell Biol 24, 509-516 (2012). chain fatty acids across the plasma membrane--lipid rafts and fatty acid transport proteins. Mol Cell Biochem 284, 135-140 (2006).

30. Bostrom P, et al. SNARE proteins mediate fusion between cytosolic lipid droplets and are

29. Harder T, Scheiffele P, Verkade P, Simons K. Lipid domain structure of the plasma membrane revealed by patching of membrane components. The Journal of cell biology 141, 929-942 (1998). implicated in insulin sensitivity. Nat Cell Biol 9, 1286-1293 (2007). 
593 31. Tansey JT, et al. Perilipin ablation results in a lean mouse with aberrant adipocyte lipolysis, enhanced leptin production, and resistance to diet-induced obesity. Proceedings of the National Academy of Sciences of the United States of America 98, 6494-6499 (2001).

32. Miyoshi $\mathrm{H}$, et al. Control of adipose triglyceride lipase action by serine 517 of perilipin A globally regulates protein kinase A-stimulated lipolysis in adipocytes. The Journal of

33. Keller $\mathrm{P}$, et al. Fat-specific protein 27 regulates storage of triacylglycerol. The Journal of biological chemistry 283, 14355-14365 (2008). biological chemistry 282, 996-1002 (2007).

35. Sun Z, et al. Perilipin1 promotes unilocular lipid droplet formation through the activation of Fsp27 in adipocytes. Nature communications 4, 1594 (2013).

36. Yuan SM, et al. Over-expression of PPAR-gamma2 gene enhances the adipogenic caspase-dependent apoptosis, and dimerization with CIDEA. Am J Physiol Endocrinol Metab 297, E1395-1413 (2009).

37. Farmer SR. Transcriptional control of adipocyte formation. Cell Metab 4, 263-273 (2006). differentiation of hemangioma-derived mesenchymal stem cells in vitro and in vivo. Oncotarget 8, 115817-115828 (2017).

40. Ghaben AL, Scherer PE. Adipogenesis and metabolic health. Nat Rev Mol Cell Biol 20,

38. Bost F, Aouadi M, Caron L, Binetruy B. The role of MAPKs in adipocyte differentiation and obesity. Biochimie 87, 51-56 (2005).

39. Aubert J, Belmonte N, Dani C. Role of pathways for signal transducers and activators of transcription, and mitogen-activated protein kinase in adipocyte differentiation. Cell Mol Life Sci 56, 538-542 (1999). 242-258 (2019). 
627 41. Green H, Meuth M. An established pre-adipose cell line and its differentiation in culture. Cell 3, 127-133 (1974).

629

630 42. Dutta-Roy AK. Cellular uptake of long-chain fatty acids: role of membrane-associated fattyacid-binding/transport proteins. Cell Mol Life Sci 57, 1360-1372 (2000).

43. Wetzel C, et al. Small-molecule inhibition of STOML3 oligomerization reverses pathological mechanical hypersensitivity. Nat Neurosci 20, 209-218 (2017).

44. Prusty D, Park BH, Davis KE, Farmer SR. Activation of MEK/ERK signaling promotes adipogenesis by enhancing peroxisome proliferator-activated receptor gamma (PPARgamma ) and C/EBPalpha gene expression during the differentiation of 3T3-L1 preadipocytes. The Journal of biological chemistry 277, 46226-46232 (2002).

45. Scioli MG, Bielli A, Gentile P, Mazzaglia D, Cervelli V, Orlandi A. The biomolecular basis of adipogenic differentiation of adipose-derived stem cells. Int J Mol Sci 15, 6517-6526 (2014).

46. Wee P, Wang Z. Epidermal Growth Factor Receptor Cell Proliferation Signaling Pathways. Cancers (Basel) 9, (2017).

48. Lajoie P, Kojic LD, Nim S, Li L, Dennis JW, Nabi IR. Caveolin-1 regulation of dynamindependent, raft-mediated endocytosis of cholera toxin-B sub-unit occurs independently of caveolae. Journal of cellular and molecular medicine 13, 3218-3225 (2009).

49. Lajoie P, Goetz JG, Dennis JW, Nabi IR. Lattices, rafts, and scaffolds: domain regulation 658 of receptor signaling at the plasma membrane. The Journal of cell biology 185, 381-385 (2009). 
660 50. Disanza A, Frittoli E, Palamidessi A, Scita G. Endocytosis and spatial restriction of cell signaling. Mol Oncol 3, 280-296 (2009).

662

51. Wilfling F, Haas JT, Walther TC, Farese RV, Jr. Lipid droplet biogenesis. Curr Opin Cell Biol 29, 39-45 (2014).

52. Gross DA, Zhan C, Silver DL. Direct binding of triglyceride to fat storage-inducing transmembrane proteins 1 and 2 is important for lipid droplet formation. Proceedings of the National Academy of Sciences of the United States of America

108, 19581-19586 (2011).

53. Wilfling F, et al. Triacylglycerol synthesis enzymes mediate lipid droplet growth by relocalizing from the ER to lipid droplets. Developmental cell 24, 384-399 (2013).

54. Walther TC, Farese RV, Jr. Lipid droplets and cellular lipid metabolism. Annu Rev Biochem

55. Yu J, Li P. The size matters: regulation of lipid storage by lipid droplet dynamics. Sci China Life Sci 60, 46-56 (2017).

56. Gao G, et al. Control of lipid droplet fusion and growth by CIDE family proteins. Biochim Biophys Acta Mol Cell Biol Lipids 1862, 1197-1204 (2017).

57. Simard JR, Pillai BK, Hamilton JA. Fatty acid flip-flop in a model membrane is faster than desorption into the aqueous phase. Biochemistry 47, 9081-9089 (2008).

58. Pohl J, Ring A, Korkmaz U, Ehehalt R, Stremmel W. FAT/CD36-mediated long-chain fatty acid uptake in adipocytes requires plasma membrane rafts. Mol Biol Cell 16, 24-31 (2005).

59. Lapatsina L, et al. Regulation of ASIC channels by a stomatin/STOML3 complex located in a mobile vesicle pool in sensory neurons. Open biology 2, 120096 (2012). 
691 60. Price MP, Thompson RJ, Eshcol JO, Wemmie JA, Benson CJ. Stomatin modulates gating 692 of acid-sensing ion channels. The Journal of biological chemistry 279, 53886-53891 (2004).

693

694 61. Muir LA, et al. Adipose tissue fibrosis, hypertrophy, and hyperplasia: Correlations with 695 diabetes in human obesity. Obesity (Silver Spring) 24, 597-605 (2016). 696

697

698 


\section{Acknowledgements}

700 The authors acknowledge Mouse Genome Arrays analysis provided by Genomics

701 Center for Clinical and Biotechnological Applications of Cancer Progression Research

702 Center, National Yang-Ming University. Genomics Center for Clinical and

703 Biotechnological Applications is supported by National Core Facility for

704 Biopharmaceuticals (NCFB), Ministry of Science and Technology. We thank the

705 Taiwan Mouse Clinic (TMC) from the Academia Sinica and Taiwan Animal Consortium

706 for technical support in metabolism related experiments. We are deeply grateful to Dr.

707 Kate Hua (NYMU), Chian-Feng Chen (NYMU), Tzuu-Shuh Jou (NTU), Jia-Fwu Shyu

708 (NDMC) and Hong-Yu Chien (TCH) for their helpful scientific suggestions; Dr. Li-Li Li

709 (LTRI, Canada) for her helpful proof-reading of the manuscript. This work was

710 supported by the Ministry of Education, Aim for the Top University Plan: MOST 109-

711 2740-B-010-002. The authors declare no conflicts of interest. NYMU: National Yang-

712 Ming University. NTU: National Taiwan University. NDMC: National Defense Medical

713 Center. TCH: Taipei City Hospital.

\section{Author contributions}

715 S.-C. Wu, W.-N. Lian, C.-Y. Tung and C.-H. Lin designed the research; S.-C. Wu

716 and Y.-M. Lio performed the research and analyzed the data; S.-C. Wu, J.-H. Lee,

717 H.-W. Liu and T.-W. Chen contributed microscopic approaches; W.-J. Lin, C.-Y.

718 Chen and C.-Y. Yang contributed materials; and S.-C. Wu, and C.- H. Lin wrote the 719 manuscript.

\section{Competing financial interests}

721 The authors declare that no competing financial interests exist. 


\section{Supplemental}

\section{Video 1}

725 The time-lapse recording under phase contrast and fluorescence microscopy of the 726 sequence of a LD-LD fusion event within a live adipocyte-like 3T3-L1 cell transfected 727 with hSTOM-RFP. Time is shown in hr:min. 
A

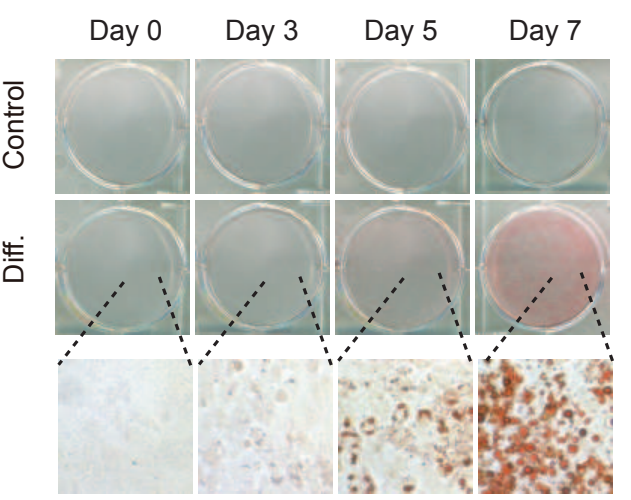

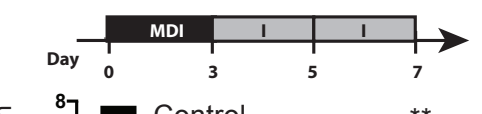

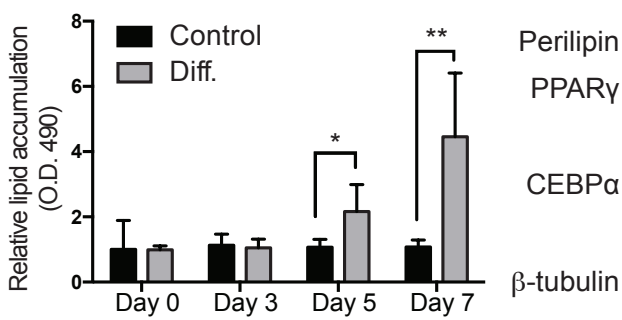

B

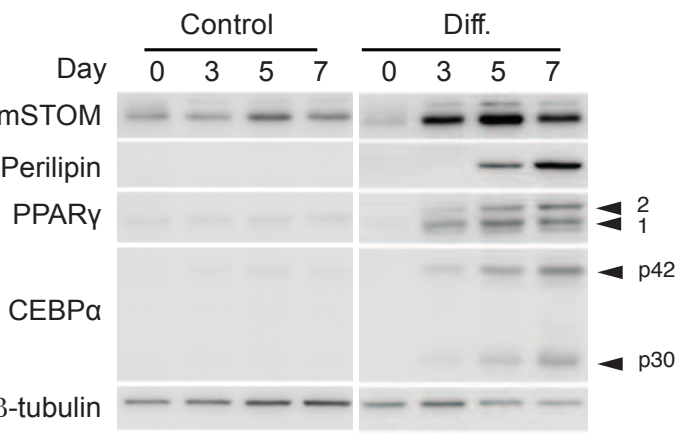

C
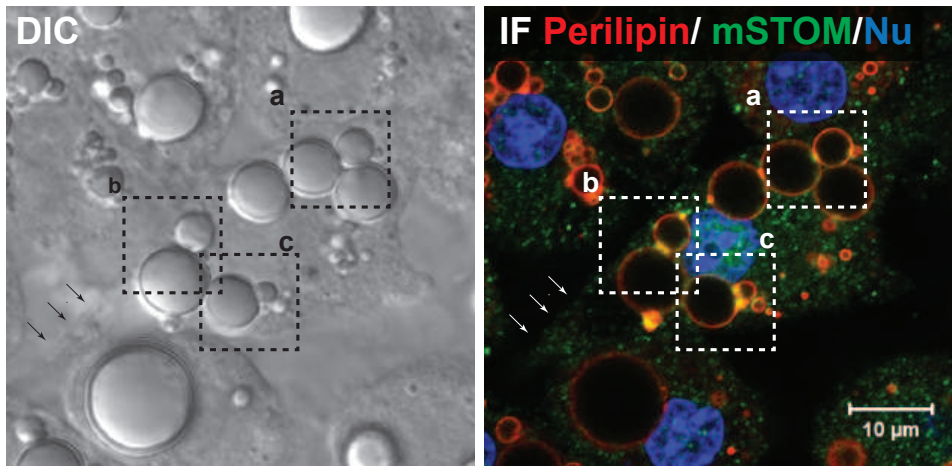

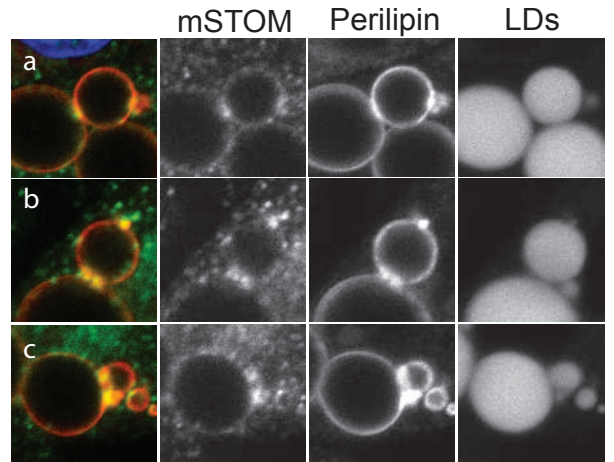

Figure 1: Stomatin expressions increased during adipogenesis. A) Mouse 3T3-L1 fibroblasts were treated with MDI induction medium (Diff.) or control vehicle. On day $0,3,5,7$ after induction, cells were stained with Oil Red $O$ and observed under microscopy. The amounts of lipid accumulation for individual culture plates were quantified by measuring absorbance at $490 \mathrm{~nm}$ (OD 490). Mean \pm s.d. for six independent experiments. ${ }^{* *} \mathrm{P}<0.01$ and ${ }^{* * *}{ }^{*}<0.0001$ by two-way ANOVA analysis. B) On the day after induction as indicated, Western blotting analyses revealed increased expressions of mouse stomatin (mSTOM), perilipin, PPARy and C/EBPa. C) Subcellular distributions of endogenous stomatin (green) and perilipin (red) on cells after 7-day induction were observed by DIC and immunofluorescence microscopy (IF). Cell nuclei were revealed by DNA stain (blue). Perilipin and mSTOM were found colocalized on vesicular membranes of lipid droplets (LDs) (inset). Bar $=10 \mu \mathrm{m}$. 

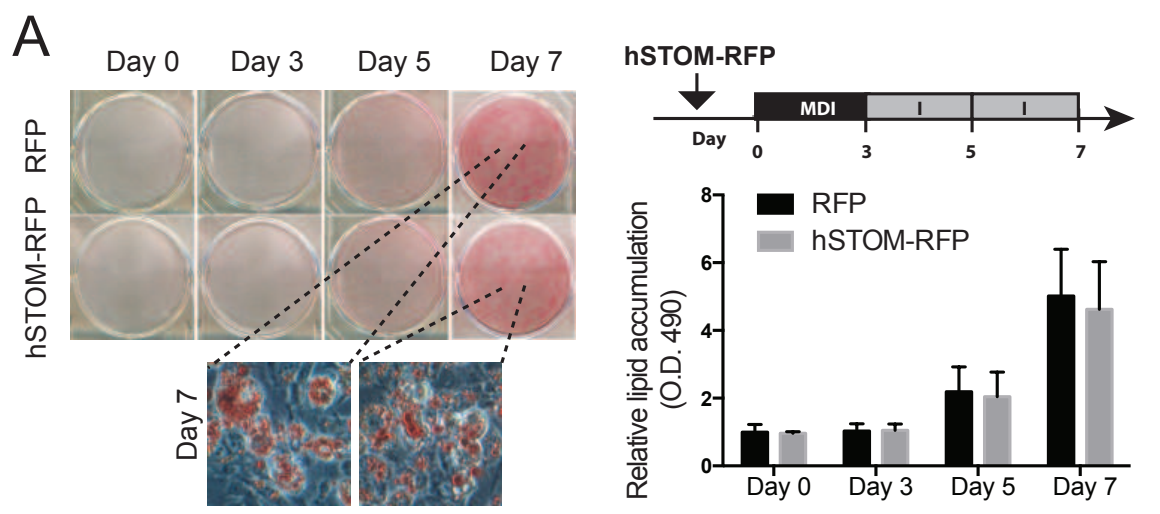

B
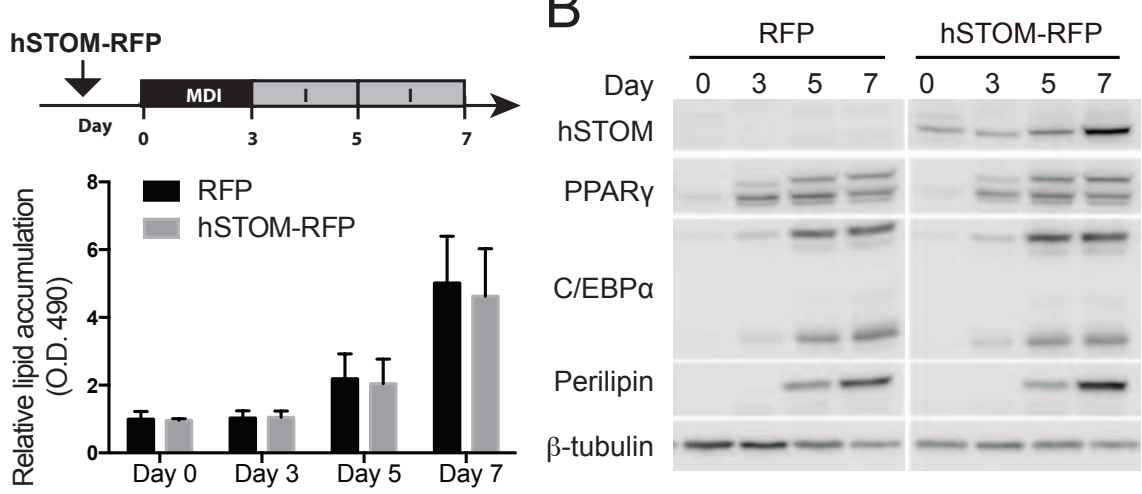

C
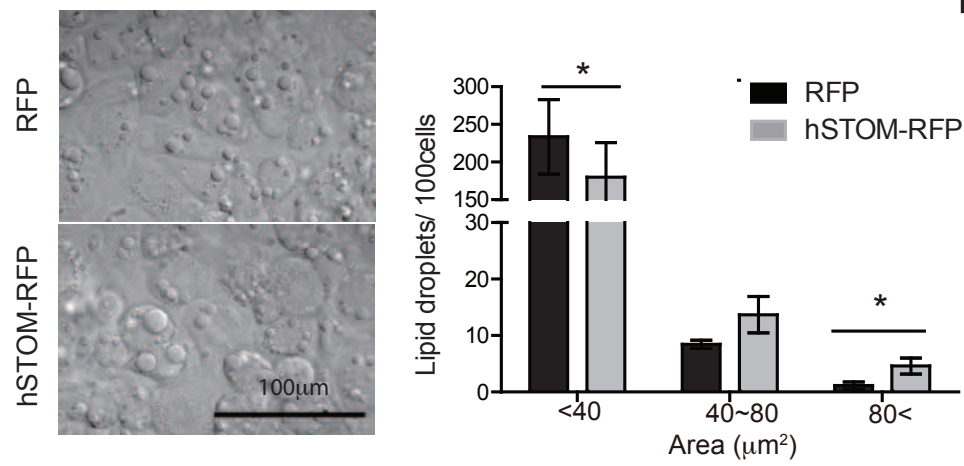

$E$
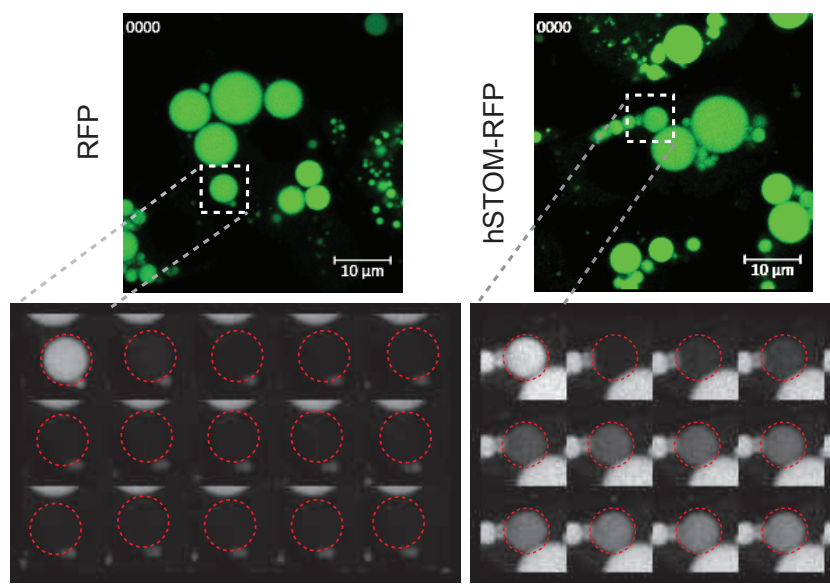

$\Delta \mathrm{T}=30 \mathrm{~s}$

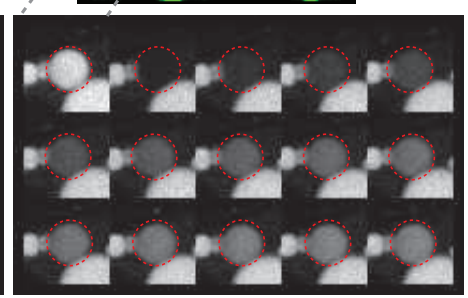

Figure 2: Overexpression of stomatin promoted lipid droplet growth and facilitated fatty acid uptake in adipocyte-like cells. A) Mouse 3T3-L1 cells over-expressing exogenous human stomatin conjugated with RFP (hSTOM-RFP) or RFP as control, were induced to differentiate into adipocyte-like cells. Lipid contents of the cells were visualized by Oil Red $\mathrm{O}$ staining and OD measured at $490 \mathrm{~nm}$. Mean \pm s.d. for four independent experiments. B) Exogenous human stomatin (hSTOM), PPARy, C/EBPa, and perilipin were examined by Western blotting. C) The numbers and sizes of lipid droplets (LDs) in adipocyte-like cells (on day 7 of induction) were quantified after AdipoRed staining and plotted as a function of LD areas. There were larger LDs (> $80 \mu \mathrm{m} 2)$ found in cells over-expressing stomatin than the control cells, which contained mostly small $(<40 \mu \mathrm{m} 2)$ LDs. Mean \pm s.e.m. for three independent experiments. ${ }^{*} P<0.05$ by paired t-test. Bar $=100 \mu \mathrm{m}$. D) The time-lapse recording of a LD-LD fusion event (arrow) within a live adipocyte-like 3T3-L1 cell transfected with hSTOM-RFP. A small LD (green dashed circle) was noted to fuse with a large LD (red dashed circle) then disappeared. Time of image tiles is shown as $\mathrm{hr}$ : min. PC: phase-contrast microscopy; Flu: fluorescence microscopy. E) Fluorescence recovery after photobleaching (FRAP) experiments were done on cells expressing hSTOM-RFP $(n=7)$ or RFP $(n=10)$. Adipocyte-like cells were pre-treated with BODIPY-FL-C12 fatty acid. Photobleaching was done at a randomly selected LD (dashed box); Afterbleaching, fluorescence recovery, as $\%$ of original intensity, was recorded by time-lapse microscopy at a 30-sec interval. Mean \pm s.e.m. for three independent experiments. Bar $=10 \mu \mathrm{m}$. F) Adipocyte-like cells expressing FLAG-conjugated human stomatin (hSF) or vector control (V) were treated with 0.2 $\mu \mathrm{M}$ fluorescently-labeled fatty acid (BODIPY-FL-C16) to measure uptake of extracellular fatty acid into the cells, or with fluorescent tag (BODIPY-FL) as a control to measure non-specific leak of BODIPY-FL into the cell. Intracellular accumulations of fluorescence over time were recorded and plotted as a function of time. Mean \pm s.e.m. for three independent experiments. 


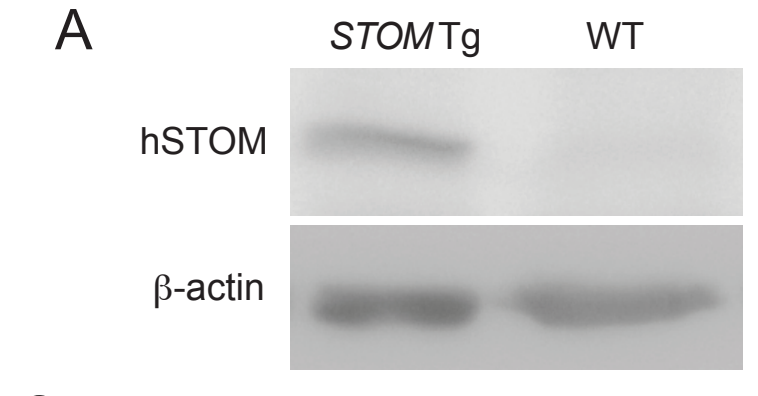

B
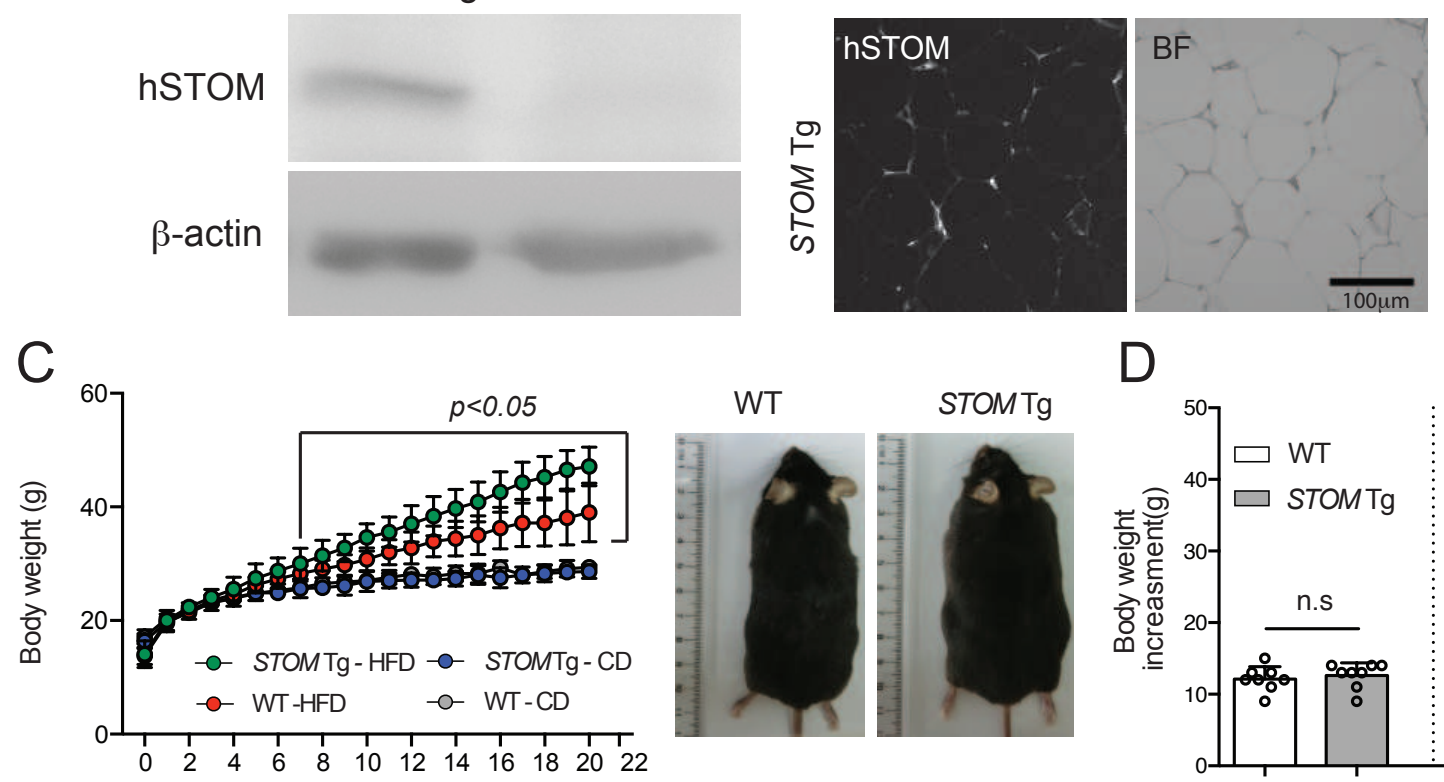

D

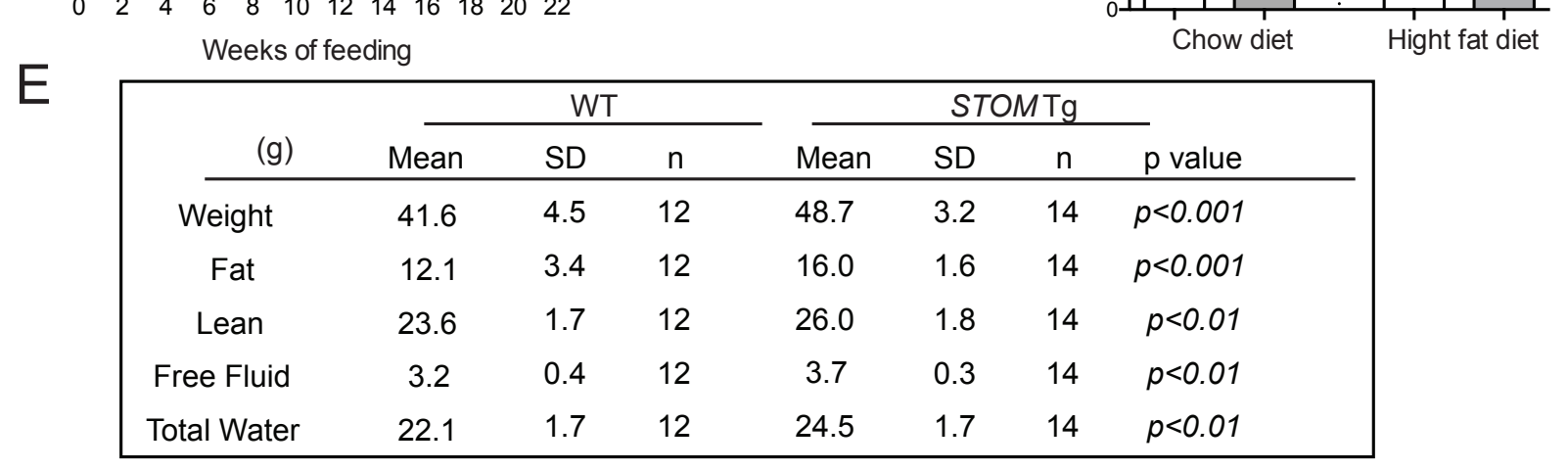
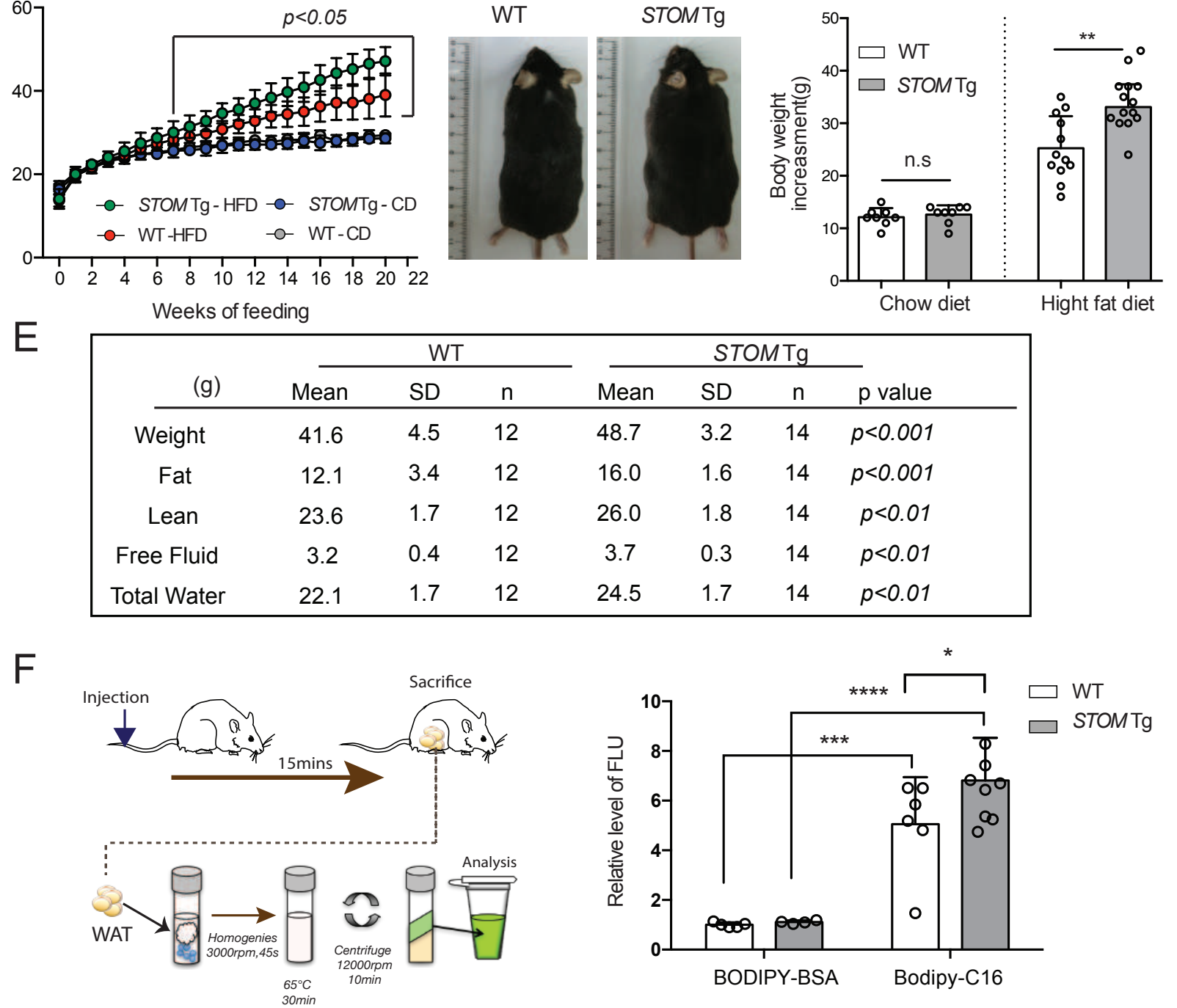

Figure 3: Stomatin transgenic mice fed with high-fat diet were more obese than the control mice. A) Western blotting revealed high expression of human stomatin (hSTOM) in stomatin transgenic mice (STOM Tg). B) The exogenous hSTOM proteins were present mainly on surface of the adipocyte plasma membrane in fat tissues of STOM Tg mice. C) Body weight changes of STOM Tg and wild type (WT) mice fed with regular chow diet (CD) or high-fat diet (HFD) for 20 weeks. Representative photos of the mice are shown. D) Body weight increments after 20-week feeding were measured. Each dot represents one mouse. While no difference was noticed in animals fed with CD, body weight gains were significantly higher in HFD-fed STOM Tg, compared with HFD-fed WT mice. Mean \pm s.d. is shown. n.s = non-significant. ${ }^{* *} P<0.01$ by unpaired t-test. E) The mass of whole body, fat, lean, free fluid, and total water were calculated for HFD-fed STOM Tg and HFD-fed WT mice. F) Fatty acid uptake was measured in vivo. Fluorescently-labeled BODIPY-FL-C16 fatty acid, or BODIPY-BSA, were injected into tail vein of STOM Tg mice or WT mice. After $15 \mathrm{~min}$, aqueous portions of white adipose tissue (WAT) from the animal were extracted and fluorescence signals that represented lipid uptake were quantified. Data shown are fold changes of fluorescence intensity using BODIPY-BSA injected to WT mice as the reference. Each dot represents one mouse. Mean \pm s.d. for three independent experiments. ${ }^{*} \mathrm{P}<0.05$, ${ }^{* *} \mathrm{P}<0.01$, and ${ }^{* * * *} \mathrm{P}<0.0001$ by two-way $A N O V A$. BODIPY-FL-C16: 4,4-Difluoro-5,7-Dimethyl-4-Bora-3a,4a-Diaza-s-Indacene-3-Hexadecanoic Acid; BSA: bovine serum albumin 

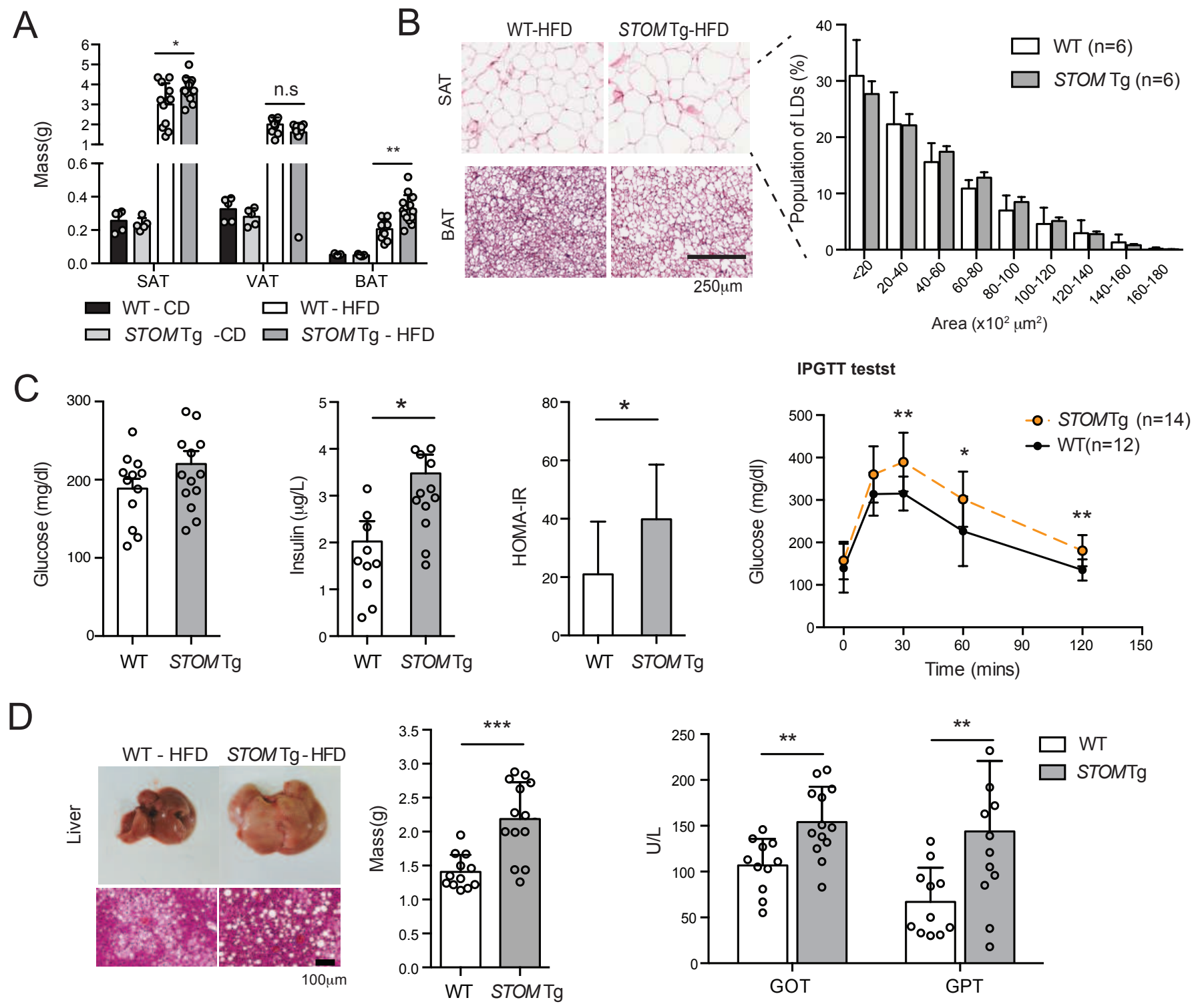

Figure 4: Stomatin transgenic mice fed with high-fat diet exhibited adipocyte hypertrophy and metabolism disorders. A) The weights of subcutaneous adipose tissue (SAT), visceral adipose tissue (VAT) and brown adipose tissue (BAT) in stomatin transgenic mice (STOM Tg) or wild type mice (WT) fed with regular chow diet (CD) or high-fat diet (HFD) for 20 weeks were measured. B) Representative H\&E stained histopathological sections of SAT, and BAT from HFD-fed STOM Tg or HFD-fed WT mice. Histogram analyses of sizes of adipocytes are shown. Bar $=250 \mu \mathrm{m}$. C) After HFD-feeding for 20 weeks, the animal's fasting glucose, serum insulin, homeostatic model assessment of insulin resistance (HOMA-IR), and intraperitoneal glucose tolerance test (IPGTT) were examined. D) The mass of liver of STOM Tg or WT mice, fed with HFD for 20 weeks, were weighed. Fatty liver changes revealed by histopathological sections and high level of serum glutamate oxaloacetate transaminase (GOT) and glutamate pyruvate transaminase (GPT) indicated impaired hepatic functions in STOM Tg mice, compared to WT mice. Bar $=100 \mu \mathrm{m}$. Each dot represents one mouse. Mean \pm s.d. is shown. $n . s=$ non-significant. ${ }^{*} P<0.05$, ${ }^{* *} P<0.01$, and ${ }^{* * *} P<0.001$ by unpaired t-test. 
A

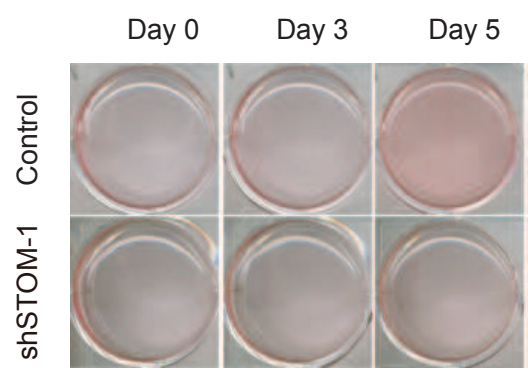

C

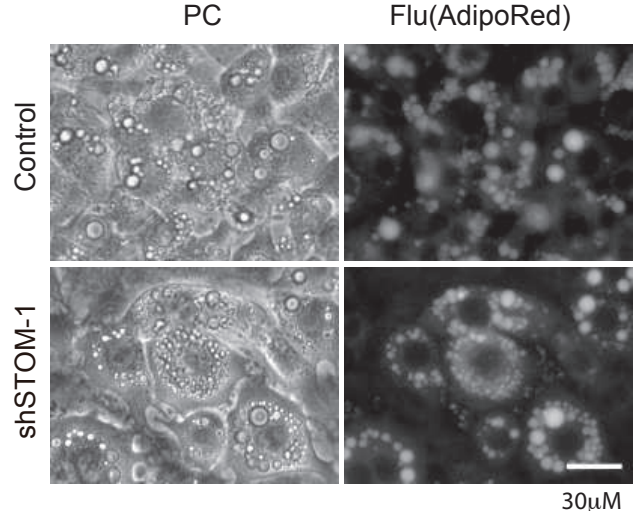

B

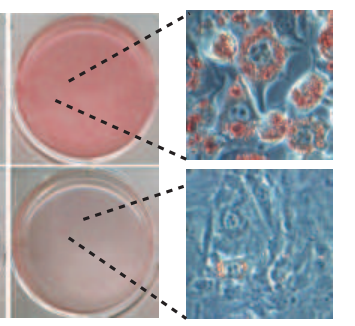

$\beta$-tubulin

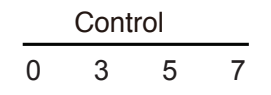

mSTOM

PPARY

CEBPa

.

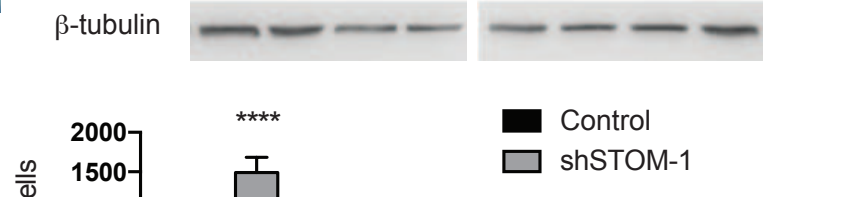

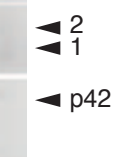

$\mathrm{p} 30$

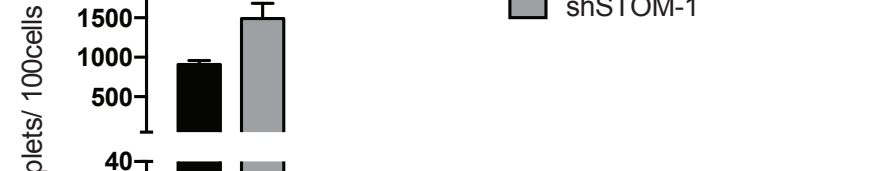

$\mathrm{D}$
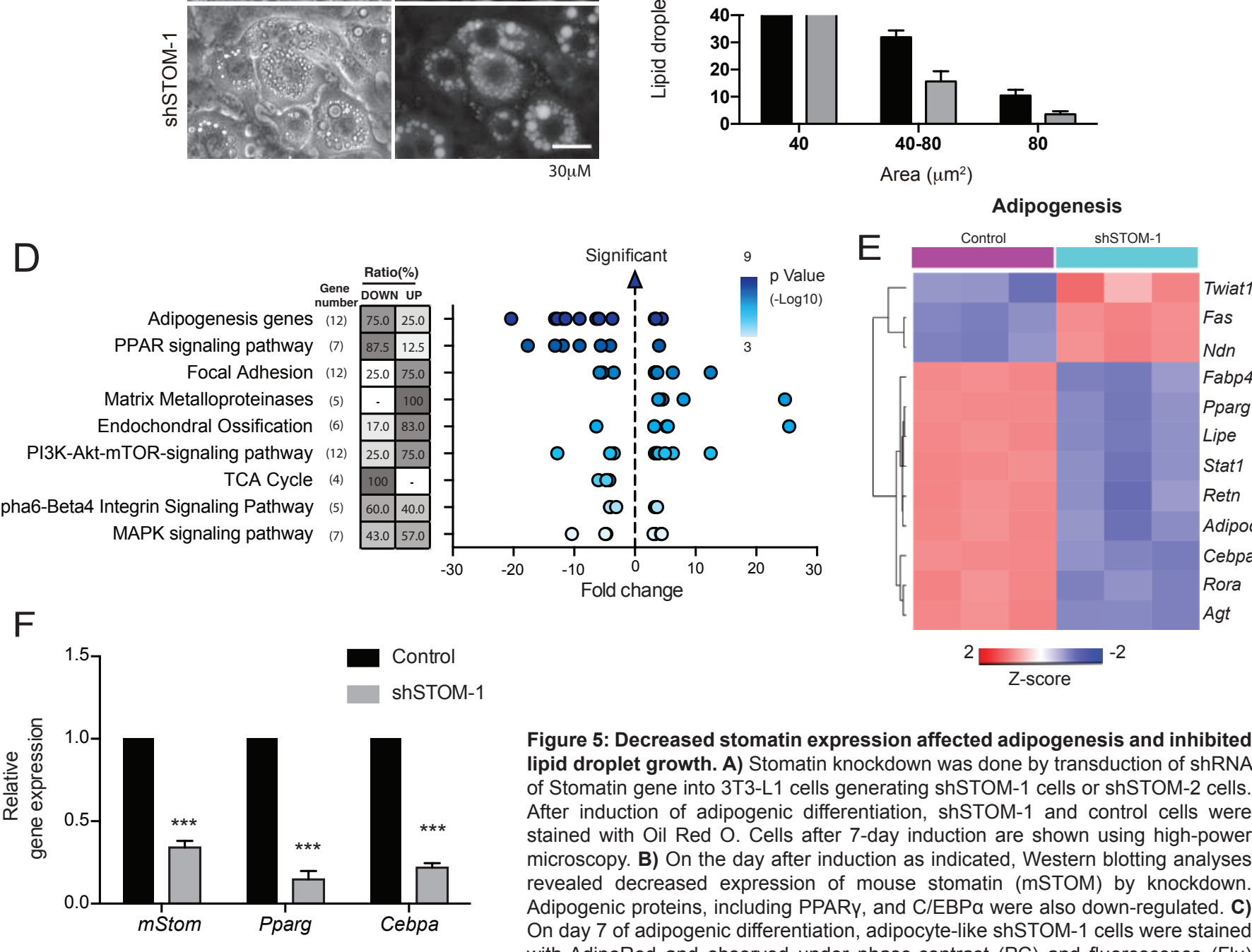

Adipogenesis

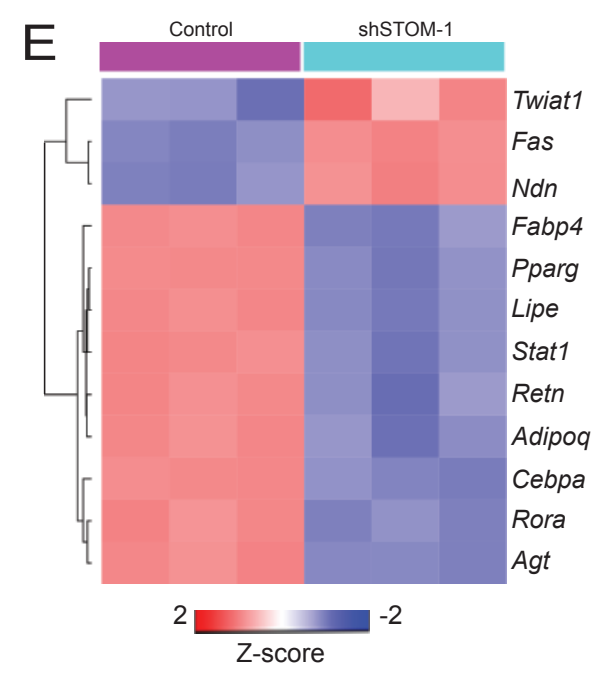

Figure 5: Decreased stomatin expression affected adipogenesis and inhibited lipid droplet growth. A) Stomatin knockdown was done by transduction of shRNA of Stomatin gene into 3T3-L1 cells generating shSTOM-1 cells or shSTOM-2 cells. After induction of adipogenic differentiation, shSTOM-1 and control cells were stained with Oil Red O. Cells after 7-day induction are shown using high-power microscopy. B) On the day after induction as indicated, Western blotting analyses revealed decreased expression of mouse stomatin (mSTOM) by knockdown. Adipogenic proteins, including PPARy, and C/EBPa were also down-regulated. C) On day 7 of adipogenic differentiation, adipocyte-like shSTOM-1 cells were stained with AdipoRed and observed under phase-contrast (PC) and fluorescence (Flu) microscopy. The size of lipid droplets (LDs) were measured, and their number per 100 adipocytes were calculated. Histogram analyses showed more small LDs $\left(<40 \mu \mathrm{m}^{2}\right)$ and fewer large $\left(>80 \mu \mathrm{m}^{2}\right)$ LDs in shSTOM-1 cells than the control. Mean $\pm \mathrm{s}$.d. for three independent experiments. ${ }^{* * * *} \mathrm{P}<0.0001$ by two-way ANOVA. Bar $=30 \mu \mathrm{m}$. D) Transcriptome analyses of adipocyte-like shSTOM-1 and control cells after induction for 7 days. From the data of microarray assays, scatter plots revealed enriched Wiki pathways in shSTOM- 1 cells comparing to the control. For a given pathway, ratio of gene number being up- or down-regulated in that pathway were determined, and plotted as function of fold change. Each dot represents one gene. The color of the dots represents the range of P-values related to the indicated pathway. E) Heat map of adipogenesis gene obtained from the enrichment-based cluster analysis of the Wiki pathway. F) Real-time GPCR analyses to validate the changed genes revealed by microarray assays. Data shown are fold changes relative to Nono mRNA level. Mean \pm s.d. for three independent experiments. ${ }^{* * *} \mathrm{P}<0.001$ by unpaired t-test. 
A

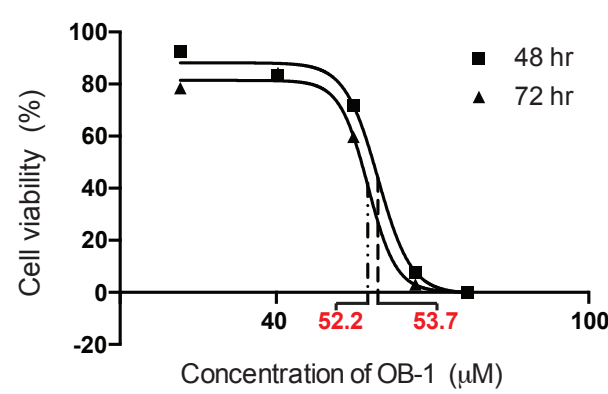

C

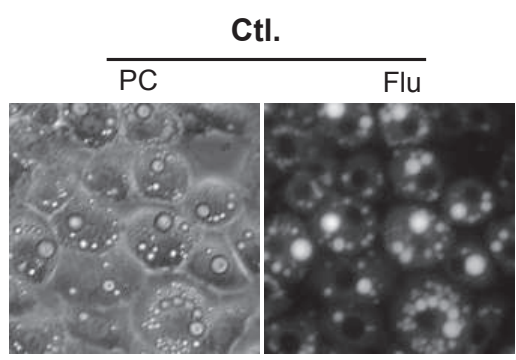

B

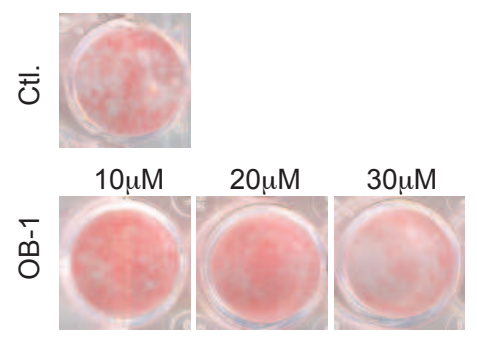

OB-1

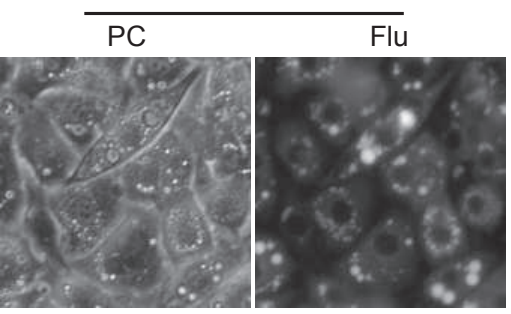

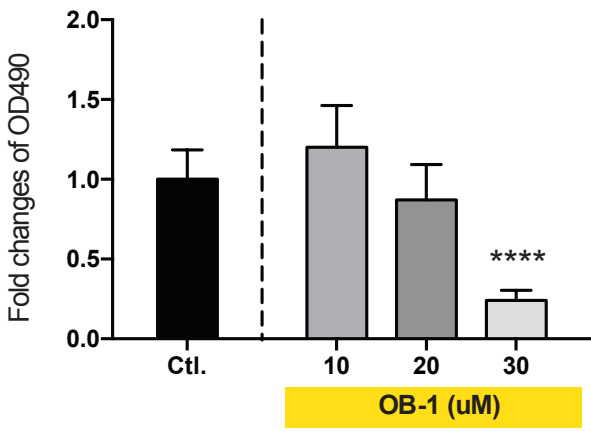

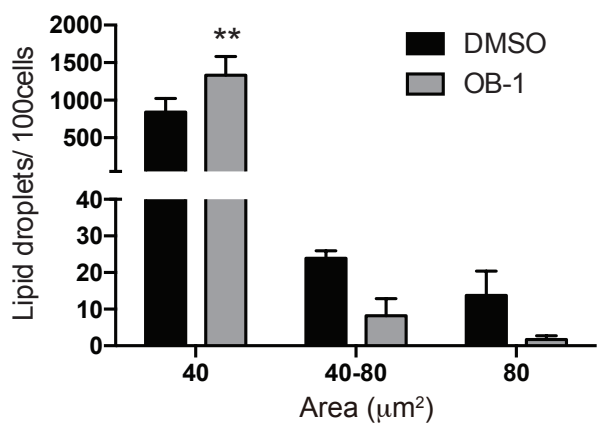

Figure 6: Stomatin inhibitor OB-1 inhibited adipogenesis and hindered lipid droplet growth. A) Cell viability after OB-1 treatments for $48 \mathrm{~h}$ or $72 \mathrm{~h}$ were tested by MTT assays. LC50 of OB-1 were calculated from three independent experiments. B) Culture adipocyte-like 3T3-L1 plates treated with or without OB-1 at concentrations indicated, were stained with Oil Red O and subjected to OD 490 quantifications. Note OB-1 inhibited adipogenesis in a dose-dependent manner, compared to the control DMSO treatment. Each OB-1 treatment data was normalized to the corresponding DMSO vehicle control; fold changes are shown. Mean \pm s.d. for three independent experiments $(n=9$ for each experiment). ${ }^{* * *} \mathrm{P}<0.0001$ by one-way ANOVA. C) Adipocyte-like 3T3-L13T3-L1 cells after 7-day induction were treated with $25 \mu \mathrm{M}$ OB-1 or control vehicle, and stained with AdipoRed. The cells were observed under phase contrast (PC) or fluorescence microscopy (Flu) to determine the numbers and sizes of LD. Histogram analyses showed increased small LDs $\left(<40 \mu \mathrm{m}^{2}\right)$ and decreased large $\left(>80 \mu \mathrm{m}^{2}\right)$ LDs after OB-1 treatments, compared to the control. Mean \pm s.d. for three independent experiments. ${ }^{* *} \mathrm{P}<0.01$ by two-way ANOVA. Bar $=$ $30 \mu \mathrm{m}$. 

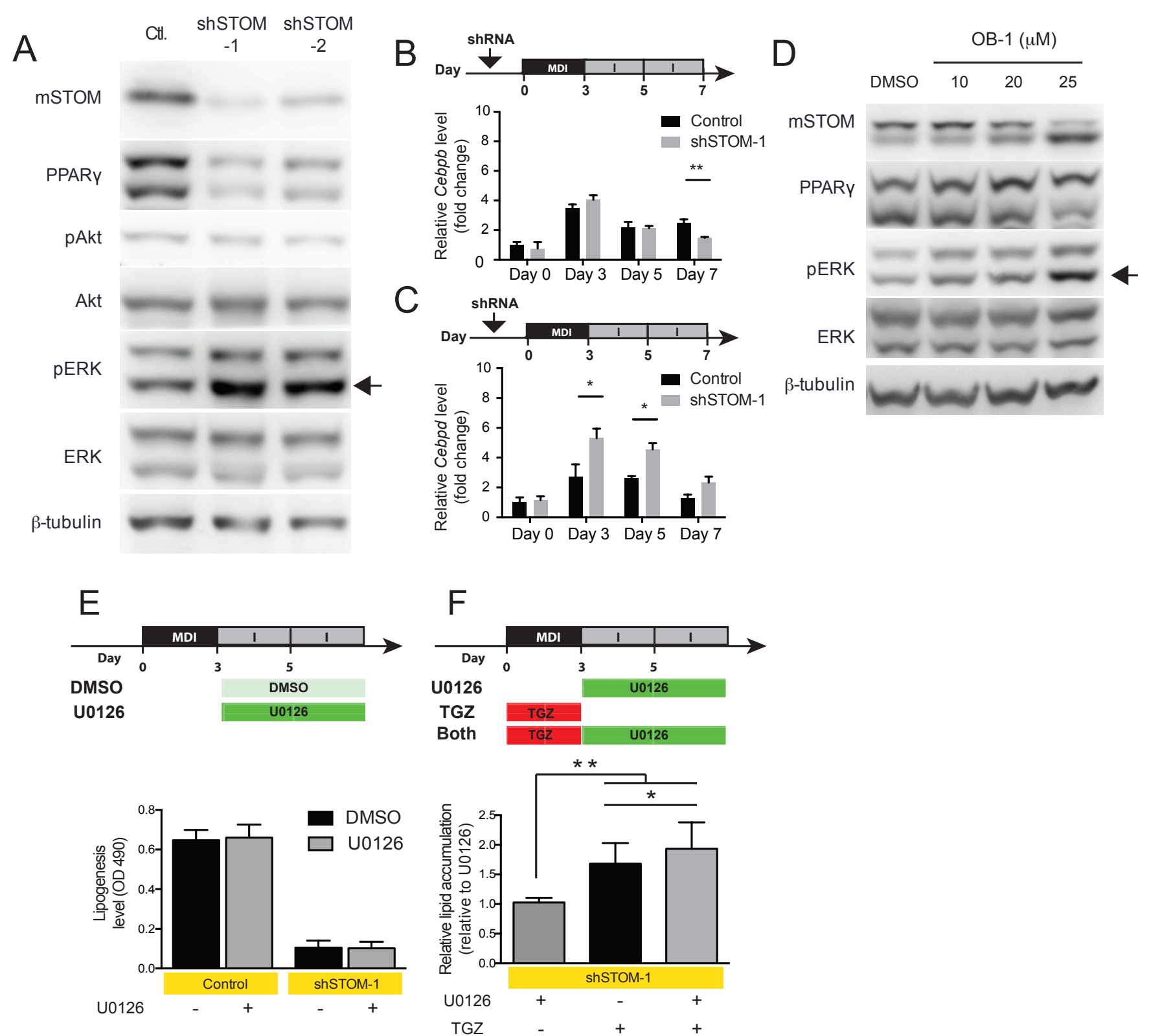

Figure 7: Knockdown of stomatin activated ERK-pathway. A) shRNA were transducted into 3T3-L1 cells to knockdown stomatin and generating shSTOM-1 and shSTOM-2 cells. After induction of differentiation, Western blotting analyses were done. Although Akt pathway remained unchanged, the ERK pathway was activated as evidenced by increased pERK (arrow). B, C) Expressions of early adipogenic genes, Cebpb and Cebpd, were examined at mRNA level by qPCR. B) Expressions of Cebpb increased in the first three days of adipogenic differentiation, and subsequently decreased. Knockdown of stomatin (exemplified by shSTOM-1 cells) did not significantly influence this pattern. C) Expressions of Cebpd gene also exhibited a transient early increase then declined; however, knockdown of stomatin appeared to significantly increase and maintain Cebpd expression at a relatively higher level than the control. The house-keeping Nono gene was used as the reference for qPCR exoeriments. ${ }^{*} \mathrm{P}<0.05$ by unpaired t-test. D) Treating adipocyte-like cells with stomatin inhibitor OB-1 also resulted in activation of ERK pathway, evidenced by increased pERK (arrow). E) Levels of lipogenesis by adipocyte-like 3T3-L1 cells were quantified by measuring OD 490 after Oil Red O staining. Treating shSTOM-1 cells with 10uM U0126, an ERK pathway inhibitor, from day3 to day 7, did not reverse the lipogenesis inhibition caused by stomatin knockdown. F) On the other hand, treating shSTOM-1cells with TGZ, a PPARY activator, in the first three days of adipogenic differentiation, was able to partially recover the knockdown-caused lipogenesis deficit; treating shSTOM-1cells with both TGZ and U0126 was noted to further increase lipid accumulation. Mean \pm s.d. for six independent experiments. ${ }^{*} \mathrm{P}<0.05$, and ${ }^{*} \mathrm{P}<0.01$ by one-way ANOVA. qPCR: quantitative real-time PCR. 


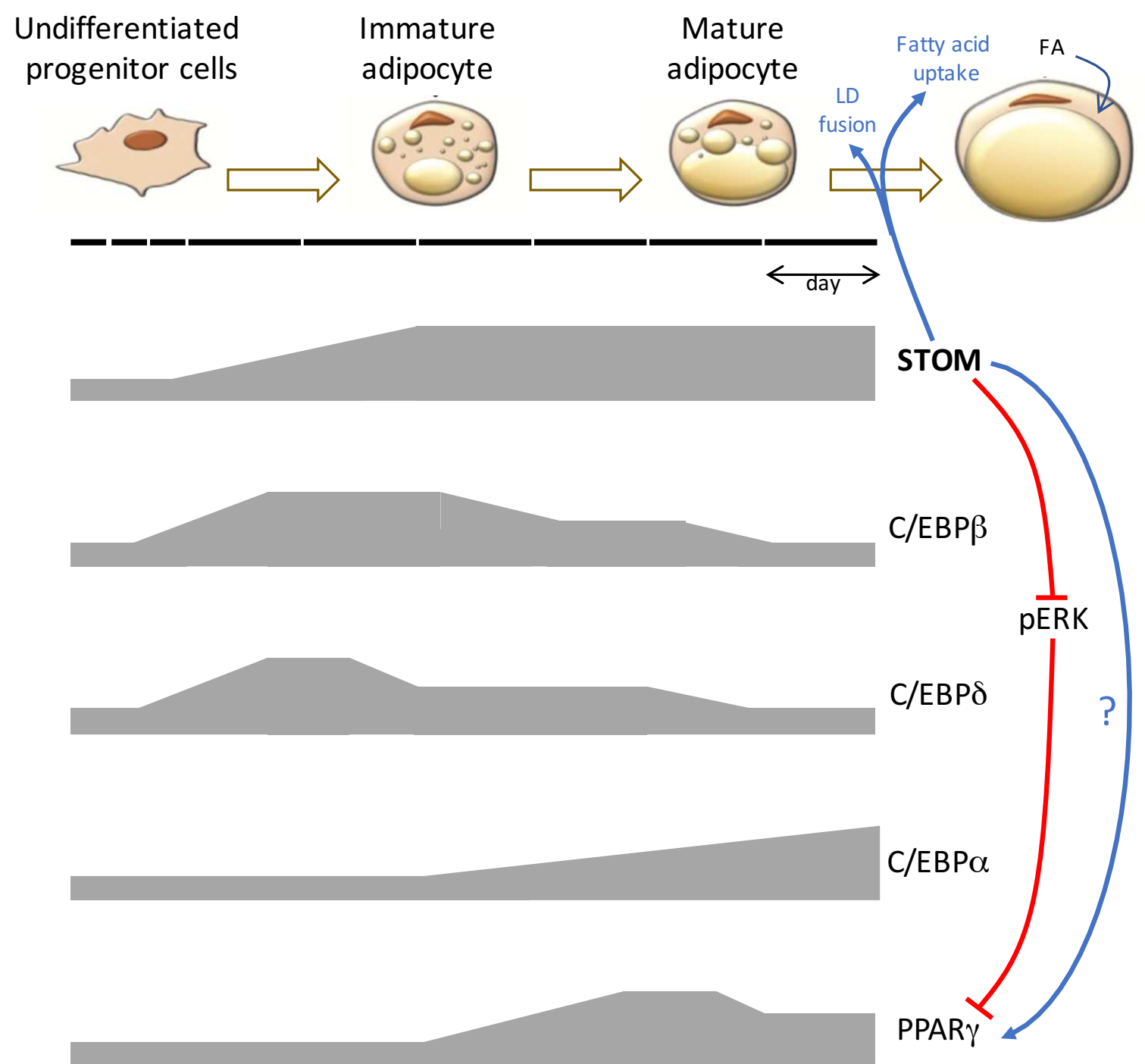

Figure 8: Stomatin's roles in modulating adipogenic differentiation and lipogenesis. Undifferentiated progenitor cells can be induced to undergo adipogenic differentiation to become immature adipocytes, and further develop into mature adipocytes, characterized by fatty acid uptake, production of lipid, and fusions and enlargements of lipid droplets (LDs). Dynamic expressions of adipogenic genes along this process are depicted. Stomatin progressively increases during adipocyte differentiation and maturation, and participates not only in fatty acid uptake and LD fusions, but also in modulation of adipogenic gene expressions. By inhibiting pERK, stomatin activates PPARY: resulting in adipocyte maturation and lipogenesis. Stomatin also plays a role in regulating early phase adipogenic genes, such as C/EBP $\beta$ and C/EBP $\delta$ through currently unknown mechanisms. C/EBP: CCAAT/enhancer binding protein; PPARY: peroxisome proliferator-activated receptor-y. 
A

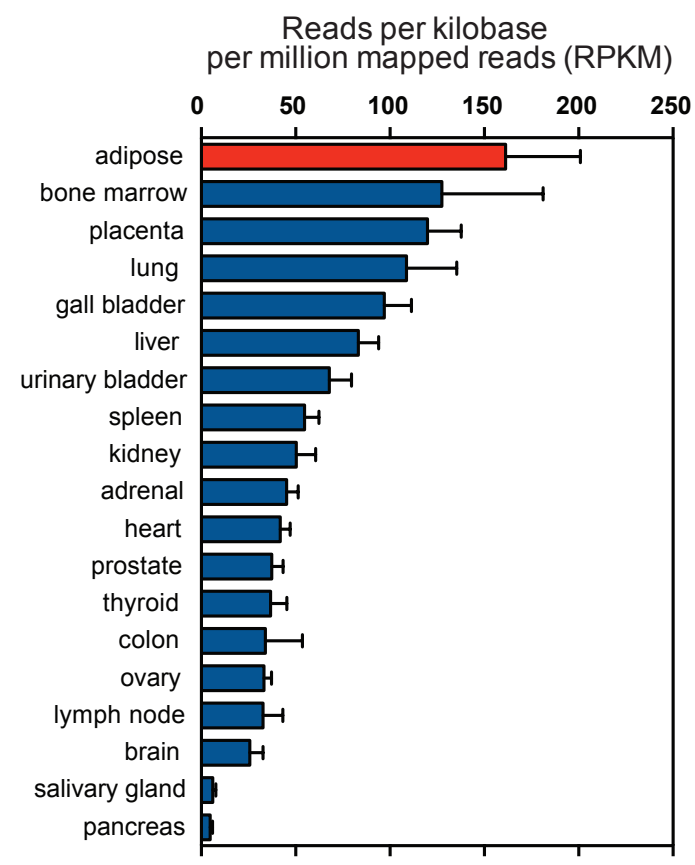

B

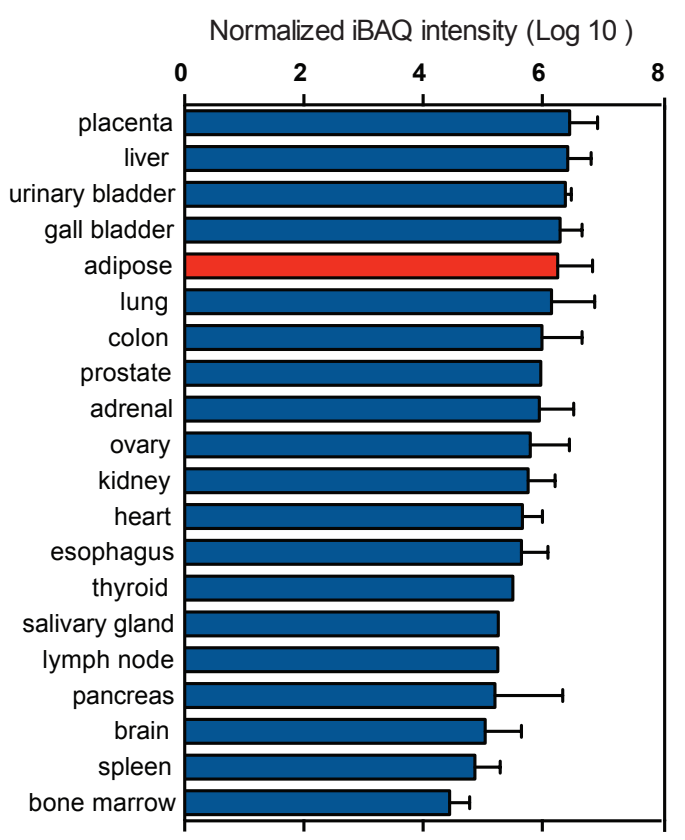

Supplemental Figure 1: In silico analyses of stomatin mRNA and proteins in different human tissues. A) The bar charts were reproduced based on the data of NCBI: https://www.ncbi.nlm.nih.gov/gene/2040? report=expression\&bioproject=PRJEB4337, containing RNA sequencing of total RNA from 20 human tissues. Adipose tissues expressed the highest amount of mRNA among all tissues examined. Mean \pm s.d. is shown. B) The bar charts were reproduced using ProteomicsDB: https://www.proteomicsdb.org/proteomicsdb/\#protein/proteinDetails/54374/expression. The error bars indicate the lowest and highest abundance level for the selected protein. 
A

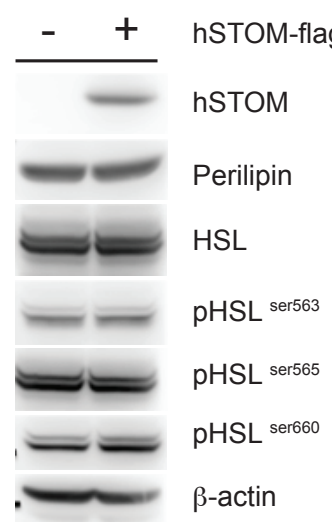

C

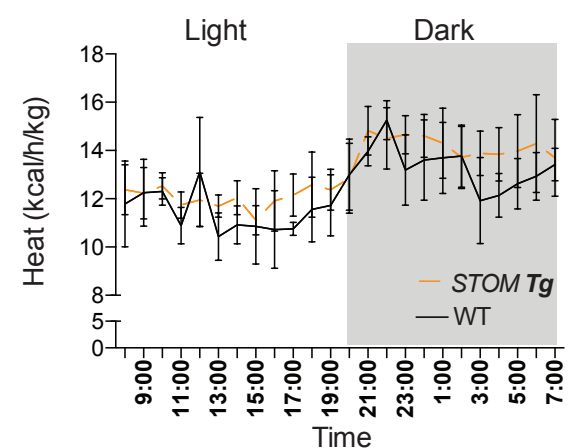

B
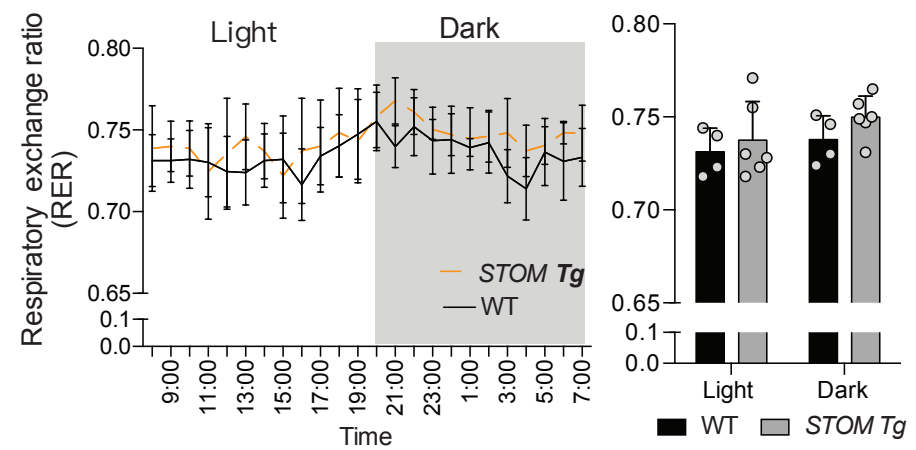
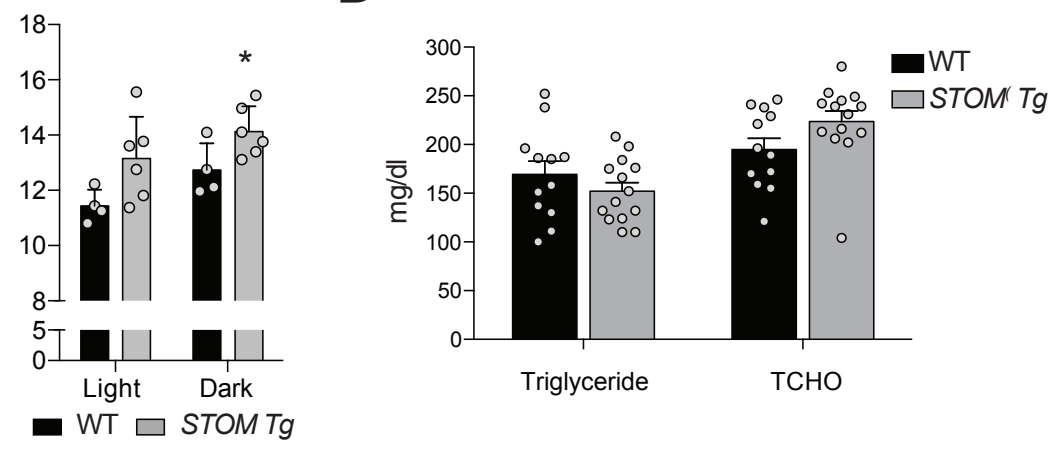

Supplemental Figure 2: Regulations of lipid metabolisms by stomatin. A) Hormone-sensitive lipase (HSL) and various phospho-HSL forms (ser563, ser565, and ser660) in adipocyte-like 3T3-L1 cells expressing human stomatin, or not, were examined by Western blotting analyses. No significant difference have been observed between overexpression of stomatin and the control. B) Energy expenditure and C) thermogenesis of STOM Tg mice and WT littermate mice after 5-month high fat diet (HFD) feeding were determined. Both measurements showed no significant difference in respiratory exchange ratio (RER) and heat production comparing STOM Tg with the control. D) Serum triglyceride and cholesterol concentrations of STOM Tg fed with HFD for 20 weeks exhibited no significant difference comparing to the control WT littermate mice. Each dot represents one mouse. Mean \pm s.d. is shown. 
A

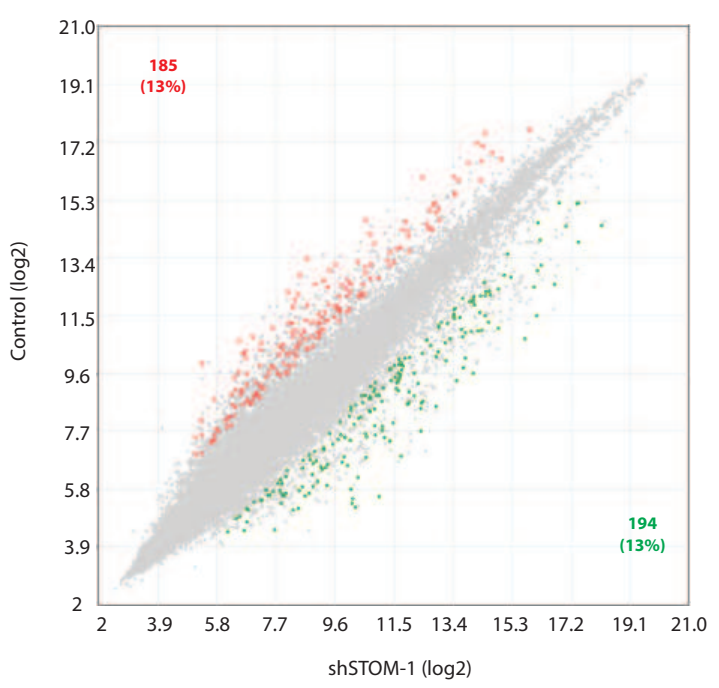

B

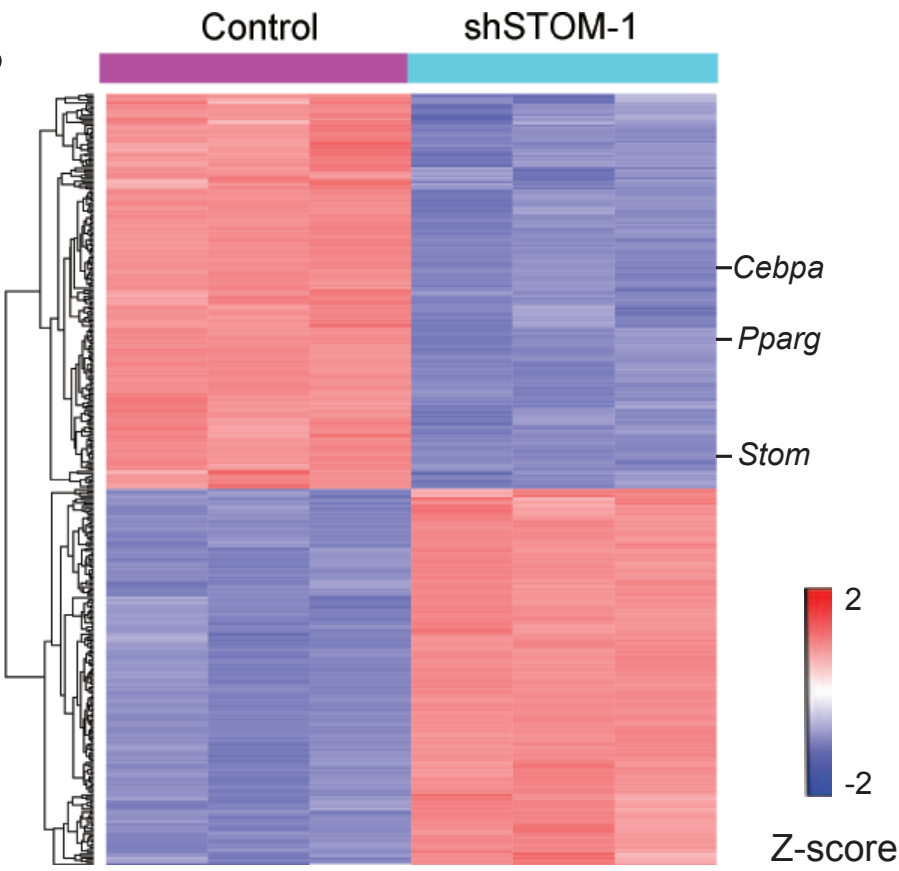

Supplemental Figure 3: Transcriptome analyses of shSTOM-1 and control cells after induction of adipogenic differentiation. A) Scatter plots of the normalized signal intensities of 1,478 genes that exhibited differential expressions between shSTOM-1 and control cells. Log2 intensities for each spot on the microarray are plotted on the $x$ and $y$ axes with signals from root tips stressed for control and shSTOM-1. The diagonal lines represent fold change cutoffs of \pm 3 . The red spots represent up-regulated genes and the green spots indicate down-regulated genes. B) Hierarchical cluster analyses of the 1,478 genes shown in A). Data were collected from three independent cell clones. Relative expression levels of genes are illustrated by the color gradient (Z-score). 


\section{Figures}
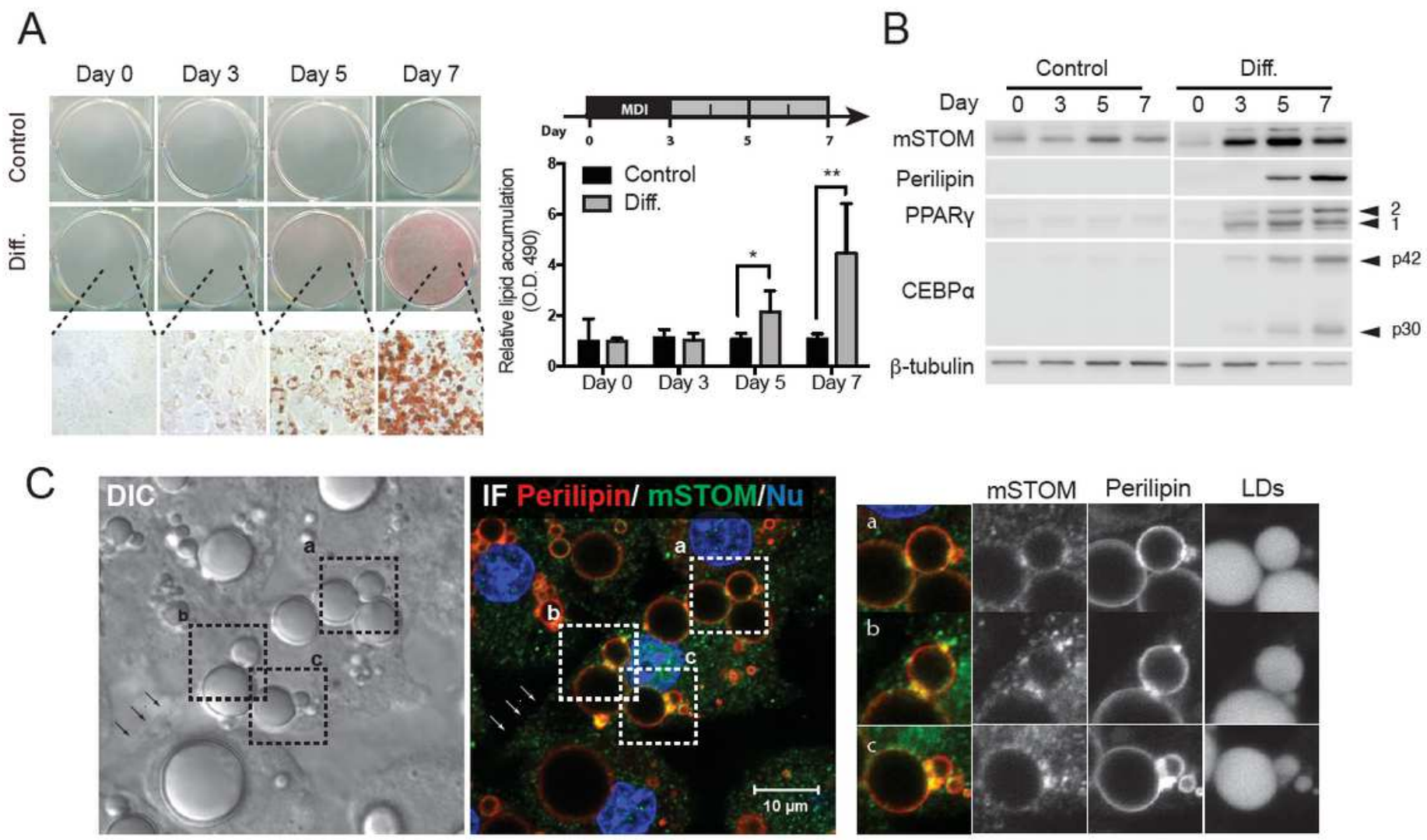

\section{Figure 1}

Stomatin expressions increased during adipogenesis. A) Mouse 3T3-L1 fibroblasts were treated with MDI induction medium (Diff.) or control vehicle. On day 0, 3, 5, 7 after induction, cells were stained with Oil Red $\mathrm{O}$ and observed under microscopy. The amounts of lipid accumulation for individual culture plates were quantified by measuring absorbance at $490 \mathrm{~nm}$ (OD 490). Mean \pm s.d. for six independent experiments. ${ }^{*} \mathrm{P}<0.01$ and ${ }^{* \star * *} \mathrm{P}<0.0001$ by two-way ANOVA analysis. $\mathrm{B}$ ) On the day after induction as indicated, Western blotting analyses revealed increased expressions of mouse stomatin (mSTOM), perilipin, PPARY and C/EBPa. C) Subcellular distributions of endogenous stomatin (green) and perilipin (red) on cells after 7-day induction were observed by DIC and immunofluorescence microscopy (IF). Cell nuclei were revealed by DNA stain (blue). Perilipin and mSTOM were found colocalized on vesicular membranes of lipid droplets (LDs) (inset). Bar $=10 \mu \mathrm{m}$. 

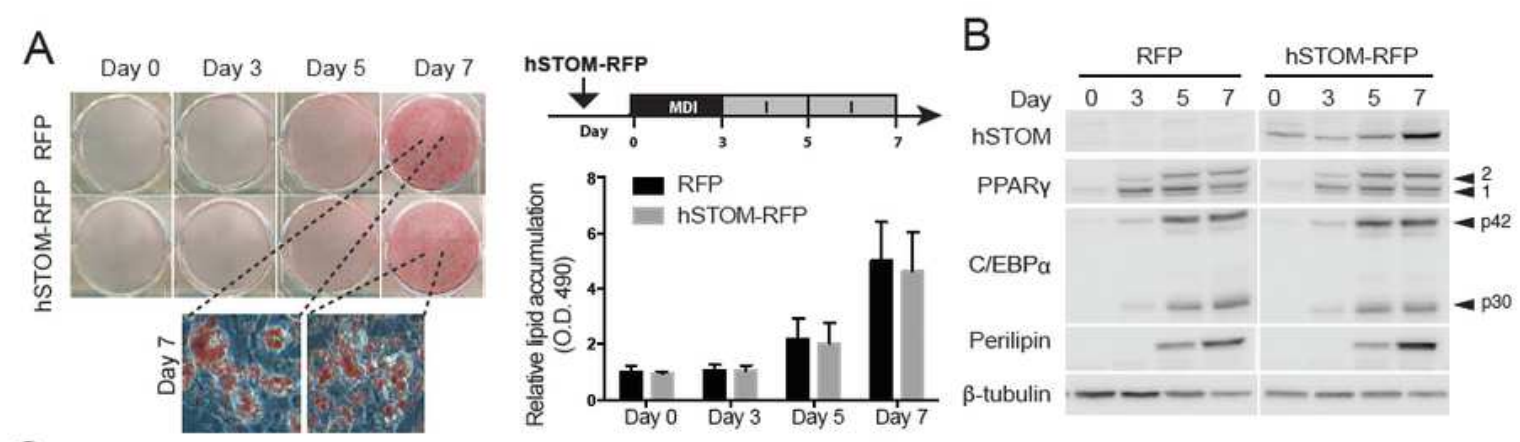

C
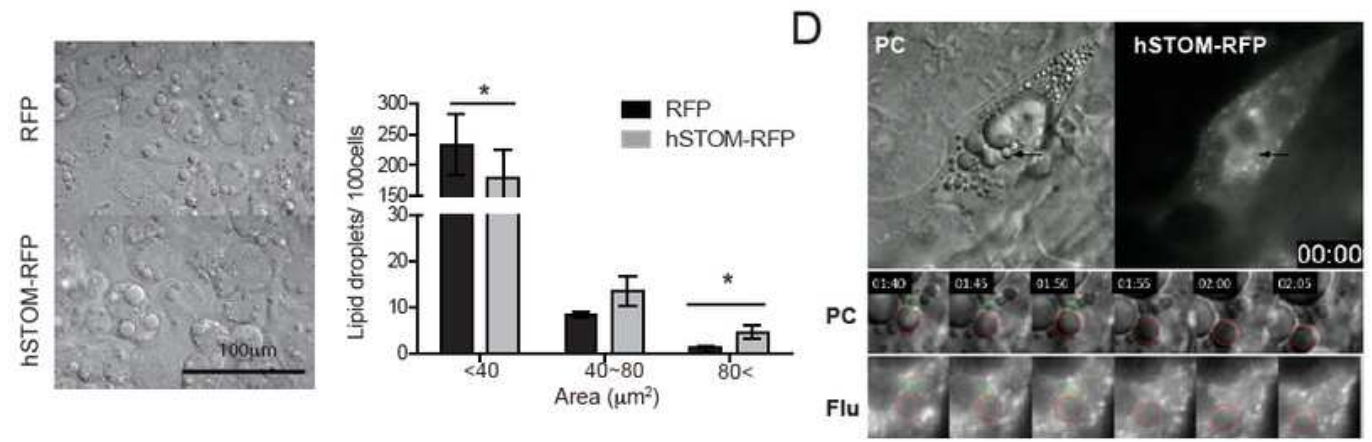

$E$
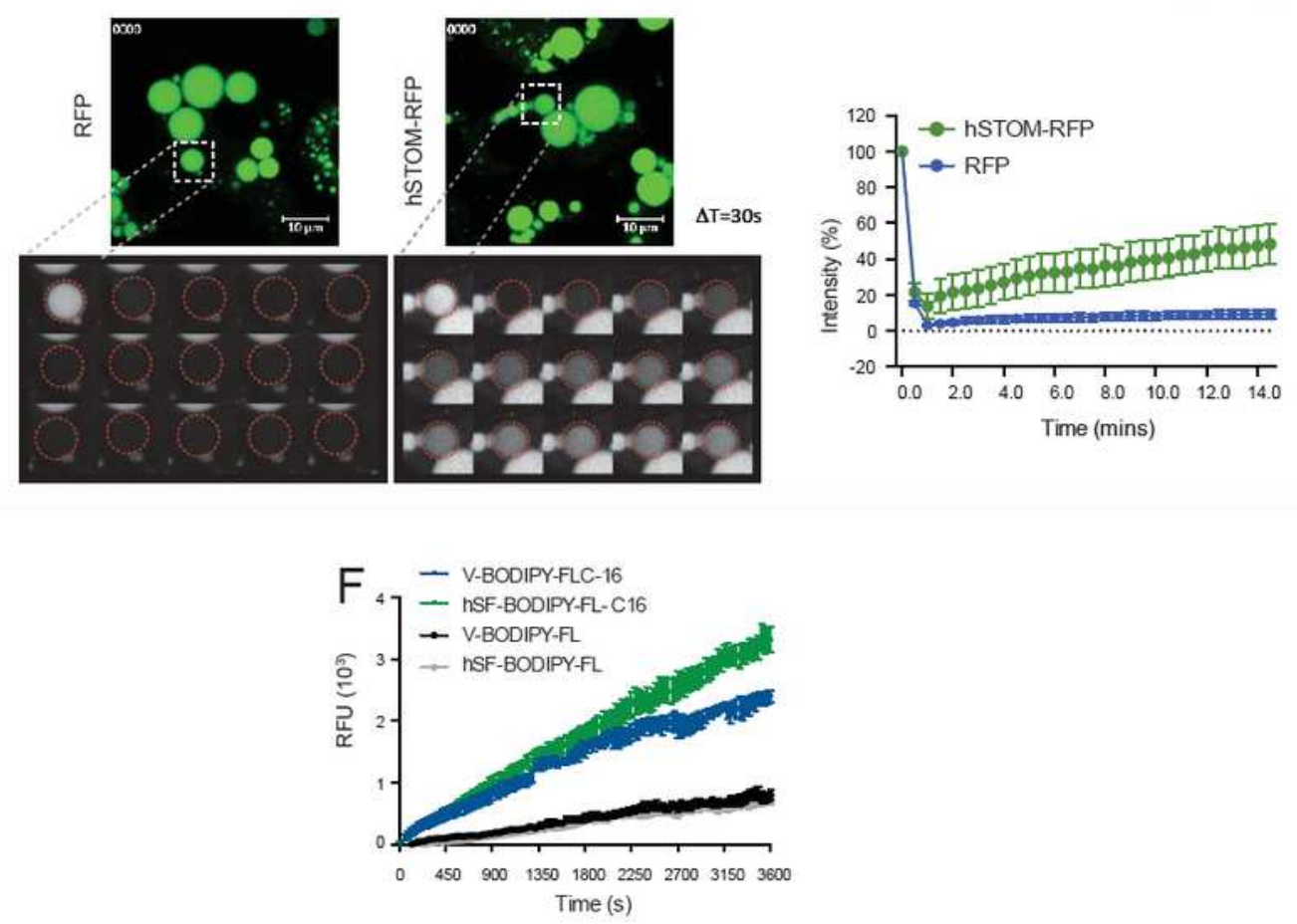

\section{Figure 2}

Overexpression of stomatin promoted lipid droplet growth and facilitated fatty acid uptake in adipocytelike cells. A) Mouse 3T3-L1 cells over-expressing exogenous human stomatin conjugated with RFP (hSTOM-RFP) or RFP as control, were induced to differentiate into adipocyte-like cells. Lipid contents of the cells were visualized by Oil Red $O$ staining and OD measured at $490 \mathrm{~nm}$. Mean \pm s.d. for four independent experiments. B) Exogenous human stomatin (hSTOM), PPARY, C/EBPa, and perilipin were 
examined by Western blotting. C) The numbers and sizes of lipid droplets (LDs) in adipocyte- like cells (on day 7 of induction) were quantified after AdipoRed staining and plotted as a function of LD areas. There were larger LDs (> $80 \mu \mathrm{m} 2)$ found in cells over-expressing stomatin than the control cells, which contained mostly small $(<40 \mu \mathrm{m} 2)$ LDs. Mean \pm s.e.m. for three independent experiments. ${ }^{*} P<0.05$ by paired t-test. $B a r=100 \mu \mathrm{m}$. D) The time-lapse recording of a LD-LD fusion event (arrow) within a live adipocyte-like 3T3-L1 cell transfected with hSTOM-RFP. A small LD (green dashed circle) was noted to fuse with a large LD (red dashed circle) then disappeared. Time of image tiles is shown as hr: min. PC: phase-contrast microscopy; Flu: fluorescence microscopy. E) Fluorescence recovery after photobleaching (FRAP) experiments were done on cells expressing hSTOM-RFP $(n=7)$ or RFP $(n=10)$. Adipocyte-like cells were pre-treated with BODIPY-FL-C12 fatty acid. Photobleaching was done at a randomly selected LD (dashed box); Afterbleaching, fluorescence recovery, as \% of original intensity, was recorded by timelapse microscopy at a 30-sec interval. Mean \pm s.e.m. for three independent experiments. Bar $=10 \mu \mathrm{m} . \mathrm{F}$ ) Adipocyte-like cells expressing FLAG-conjugated human stomatin (hSF) or vector control (V) were treated with $0.2 \mu \mathrm{M}$ fluorescently-labeled fatty acid (BODIPY-FL-C16) to measure uptake of extracellular fatty acid into the cells, or with fluorescent tag (BODIPY-FL) as a control to measure non-specific leak of BODIPY-FL into the cell. Intracellular accumulations of fluorescence over time were recorded and plotted as a function of time. Mean \pm s.e.m. for three independent experiments. 


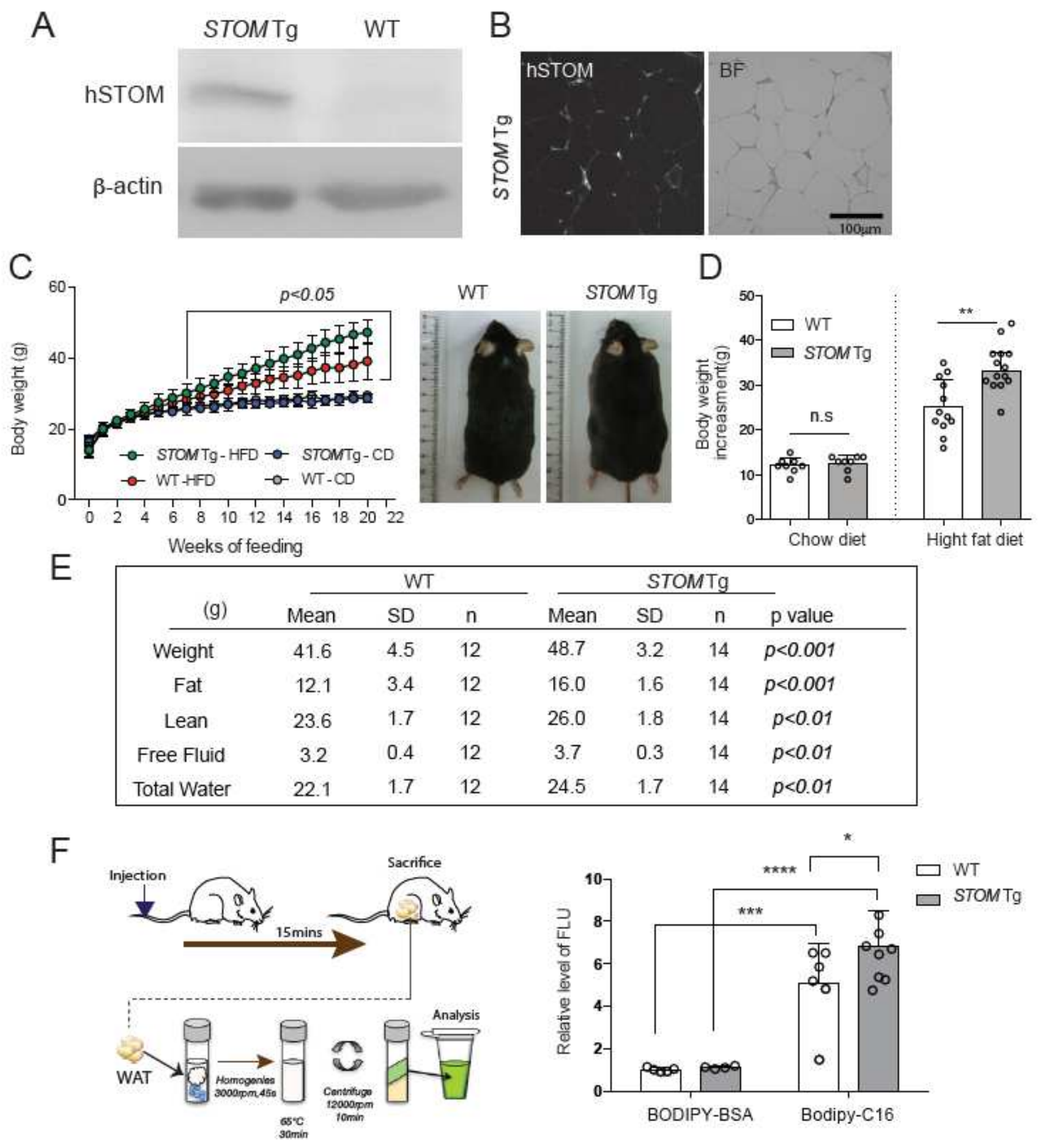

Figure 3

Stomatin transgenic mice fed with high-fat diet were more obese than the control mice. A) Western blotting revealed high expression of human stomatin (hSTOM) in stomatin transgenic mice (STOM Tg). B) The exogenous hSTOM proteins were present mainly on surface of the adipocyte plasma membrane in fat tissues of STOM Tg mice. C) Body weight changes of STOM Tg and wild type (WT) mice fed with regular chow diet (CD) or high-fat diet (HFD) for 20 weeks. Representative photos of the mice are shown. D) Body weight increments after 20-week feeding were measured. Each dot represents one mouse. While 
no difference was noticed in animals fed with CD, body weight gains were significantly higher in HFD-fed STOM Tg, compared with HFD-fed WT mice. Mean \pm s.d. is shown. $n . s=$ non-significant. ${ }^{*} P<0.01$ by unpaired t-test. E) The mass of whole body, fat, lean, free fluid, and total water were calculated for HFDfed STOM Tg and HFD-fed WT mice. F) Fatty acid uptake was measured in vivo. Fluorescently-labeled BODIPY-FL-C16 fatty acid, or BODIPY-BSA, were injected into tail vein of STOM Tg mice or WT mice. After $15 \mathrm{~min}$, aqueous portions of white adipose tissue (WAT) from the animal were extracted and fluorescence signals that represented lipid uptake were quantified. Data shown are fold changes of fluorescence intensity using BODIPY-BSA injected to WT mice as the reference. Each dot represents one mouse. Mean \pm s.d. for three independent experiments. ${ }^{*} P<0.05$, ${ }^{*} P<0.01$, and ${ }^{*} * \star * P<0.0001$ by two-way ANOVA. BODIPY-FL-C16: 4,4-Difluoro-5,7-Dimethyl-4-Bora-3a,4a-Diaza-s-Indacene-3-Hexadecanoic Acid; BSA: bovine serum albumin
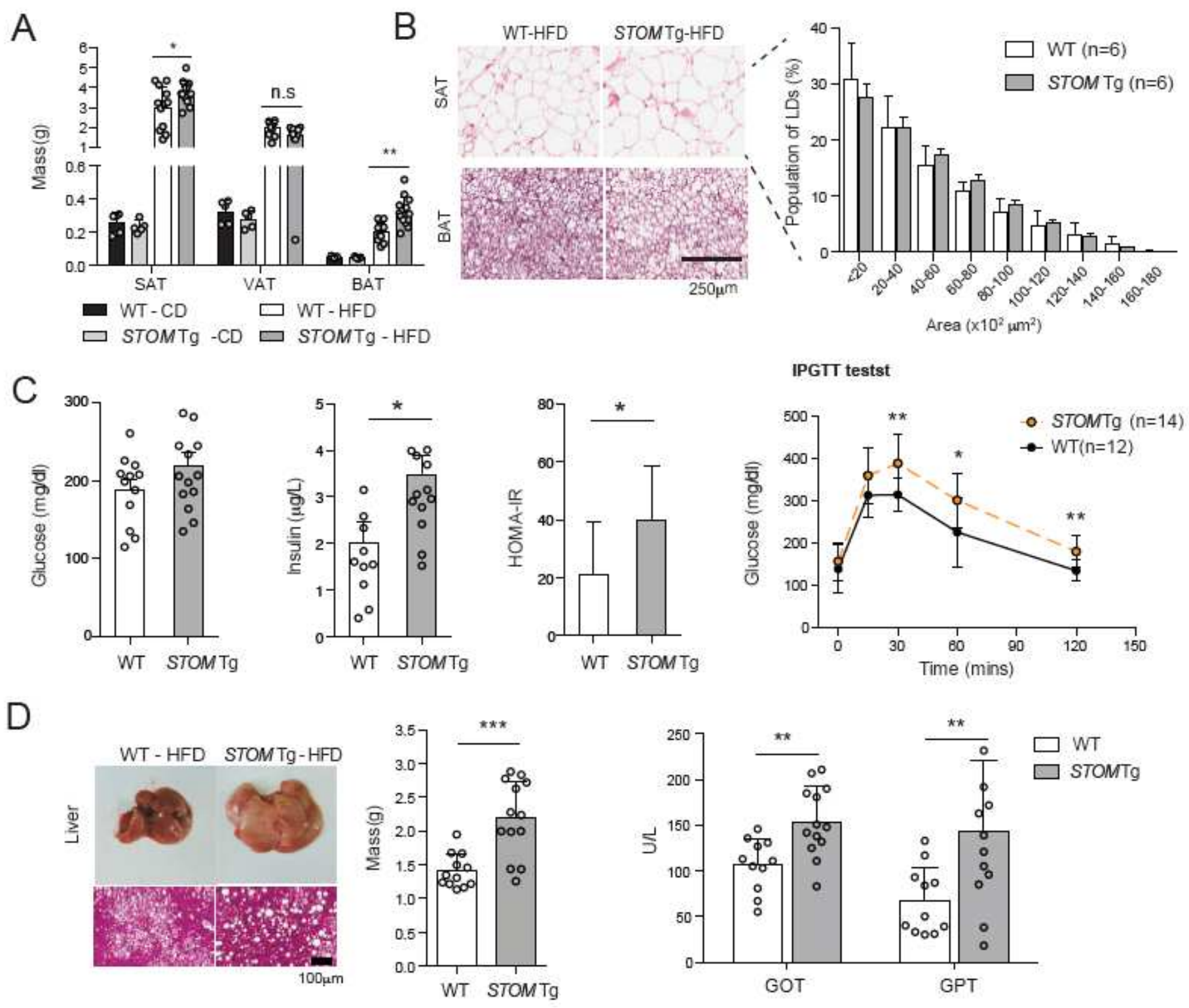

Figure 4 
Stomatin transgenic mice fed with high-fat diet exhibited adipocyte hypertrophy and metabolism disorders. A) The weights of subcutaneous adipose tissue (SAT), visceral adipose tissue (VAT) and brown adipose tissue (BAT) in stomatin transgenic mice (STOM Tg) or wild type mice (WT) fed with regular chow diet (CD) or high-fat diet (HFD) for 20 weeks were measured. B) Representative H\&E stained histopathological sections of SAT, and BAT from HFD-fed STOM Tg or HFD-fed WT mice. Histogram analyses of sizes of adipocytes are shown. Bar $=250 \mu \mathrm{m}$. C) After HFD-feeding for 20 weeks, the animal's fasting glucose, serum insulin, homeostatic model assessment of insulin resistance (HOMA-IR), and intraperitoneal glucose tolerance test (IPGTT) were examined. D) The mass of liver of STOM Tg or WT mice, fed with HFD for 20 weeks, were weighed. Fatty liver changes revealed by histopathological sections and high level of serum glutamate oxaloacetate transaminase (GOT) and glutamate pyruvate transaminase (GPT) indicated impaired hepatic functions in STOM Tg mice, compared to WT mice. Bar = $100 \mu \mathrm{m}$. Each dot represents one mouse. Mean \pm s.d. is shown. $n . s=$ non-significant. ${ }^{*} P<0.05,{ }^{*} P<0.01$, and $* \star * P<0.001$ by unpaired t-test. 

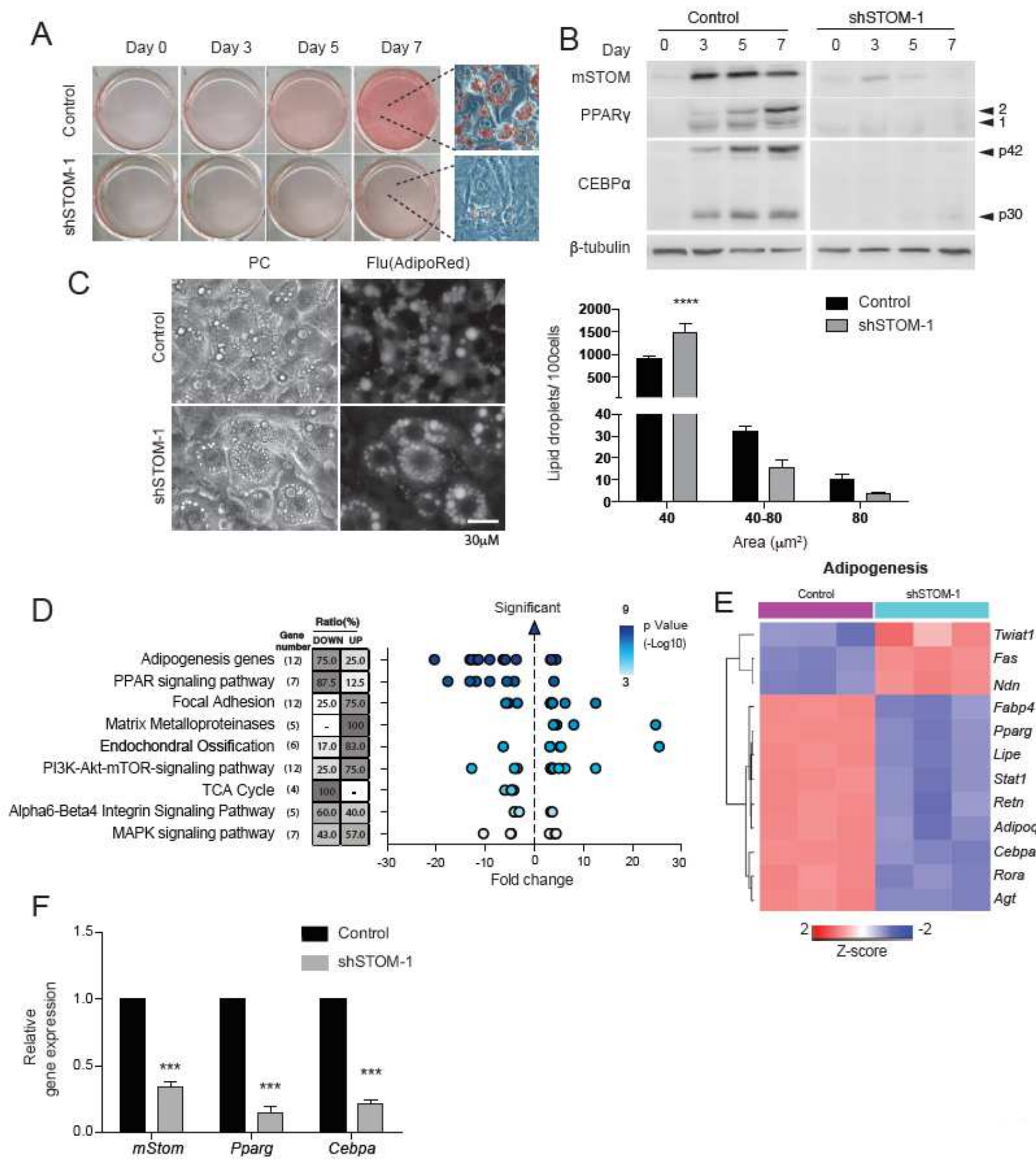

Adipogenesis

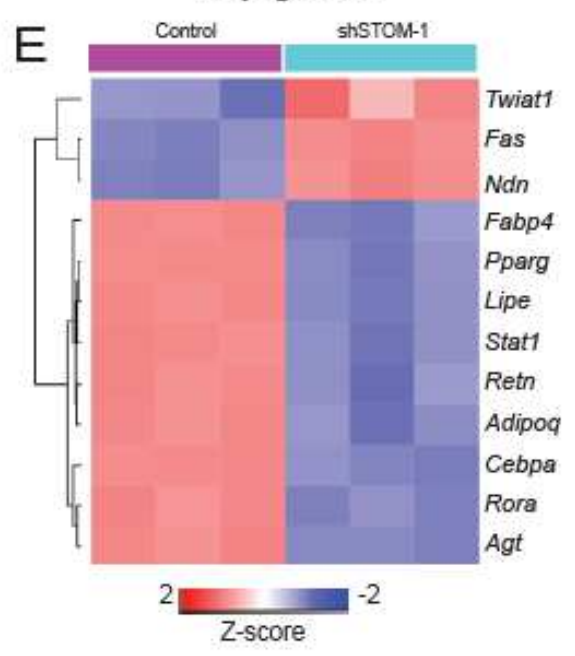

\section{Figure 5}

Decreased stomatin expression affected adipogenesis and inhibited lipid droplet growth. A) Stomatin knockdown was done by transduction of shRNA of Stomatin gene into 3T3-L1 cells generating shSTOM1 cells or shSTOM- 2 cells. After induction of adipogenic differentiation, shSTOM- 1 and control cells were stained with Oil Red 0. Cells after 7-day induction are shown using high-power microscopy. B) On the day after induction as indicated, Western blotting analyses revealed decreased expression of mouse stomatin 
(mSTOM) by knockdown. Adipogenic proteins, including PPARy, and C/EBPa were also down-regulated. C) On day 7 of adipogenic differentiation, adipocyte-like shSTOM-1 cells were stained with AdipoRed and observed under phase-contrast (PC) and fluorescence (Flu) microscopy. The size of lipid droplets (LDs) were measured, and their number per 100 adipocytes were calculated. Histogram analyses showed more small LDs $(<40 \mu \mathrm{m} 2)$ and fewer large $(>80 \mu \mathrm{m} 2)$ LDs in shSTOM-1 cells than the control. Mean \pm s.d. for three independent experiments. ${ }^{* \star * *} P<0.0001$ by two-way ANOVA. Bar $=30 \mu \mathrm{m}$. D) Transcriptome analyses of adipocyte-like shSTOM-1 and control cells after induction for 7 days. From the data of microarray assays, scatter plots revealed enriched Wiki pathways in shSTOM-1 cells comparing to the control. For a given pathway, ratio of gene number being up- or down-regulated in that pathway were determined, and plotted as function of fold change. Each dot represents one gene. The color of the dots represents the range of P-values related to the indicated pathway. E) Heat map of adipogenesis gene obtained from the enrichment-based cluster analysis of the Wiki pathway. F) Real-time qPCR analyses to validate the changed genes revealed by microarray assays. Data shown are fold changes relative to Nono mRNA level. Mean \pm s.d. for three independent experiments. ${ }^{\star * *} \mathrm{P}<0.001$ by unpaired t-test.

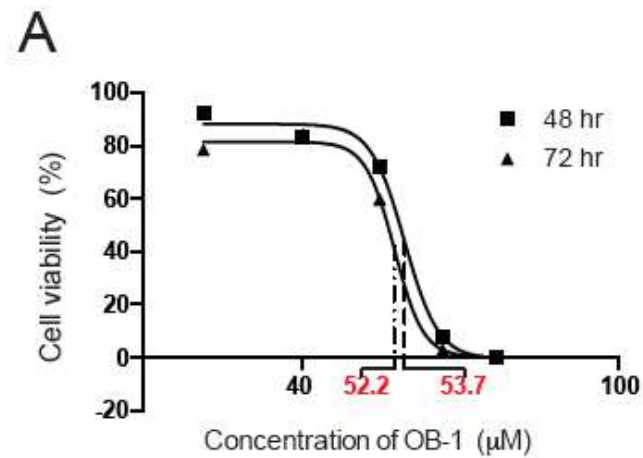

C

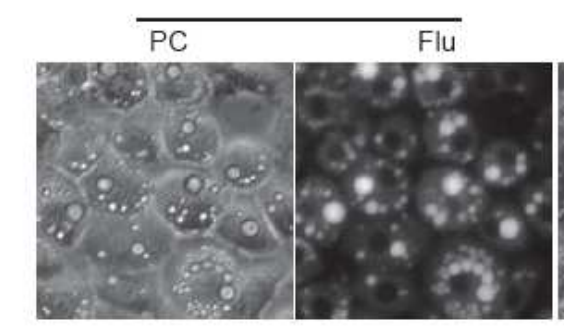

B

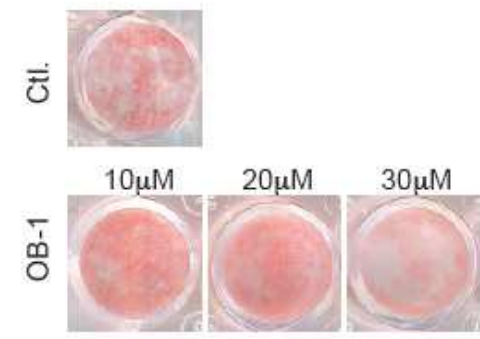

OB-1
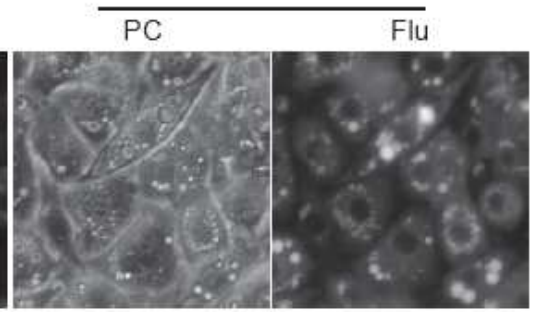
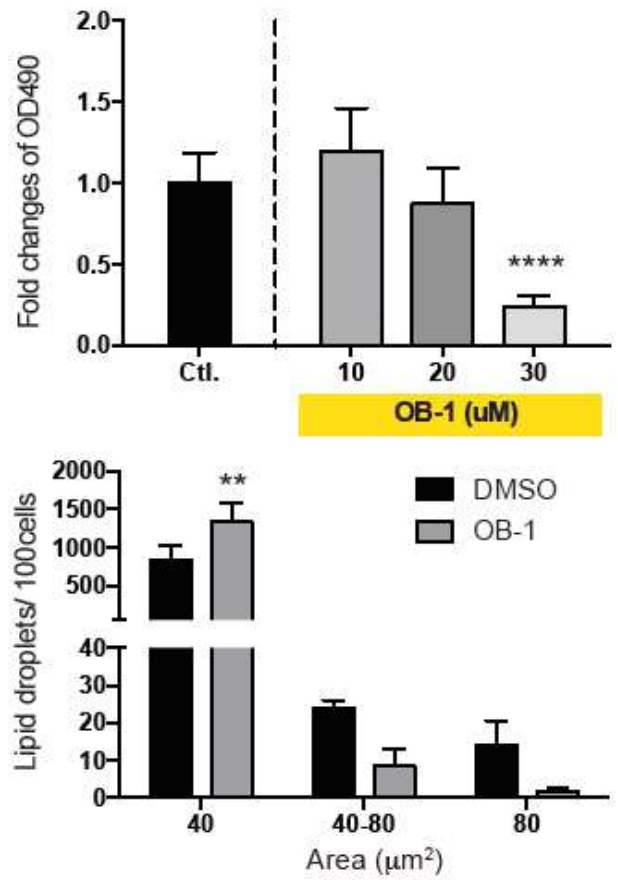

\section{Figure 6}

Stomatin inhibitor OB-1 inhibited adipogenesis and hindered lipid droplet growth. A) Cell viability after OB-1 treatments for $48 \mathrm{~h}$ or $72 \mathrm{~h}$ were tested by MTT assays. LC50 of OB-1 were calculated from three independent experiments. B) Culture adipocyte-like 3T3-L1 plates treated with or without OB-1 at concentrations indicated, were stained with Oil Red O and subjected to OD 490 quantifications. Note OB-1 inhibited adipogenesis in a dose-dependent manner, compared to the control DMSO treatment. Each OB-1 treatment data was normalized to the corresponding DMSO vehicle control; fold changes are shown. Mean \pm s.d. for three independent experiments ( $n=9$ for each experiment). ${ }^{\star \star \star *} P<0.0001$ by one-way 
ANOVA. C) Adipocyte-like 3T3-L13T3-L1 cells after 7-day induction were treated with 25 $\mu \mathrm{M}$ OB-1 or control vehicle, and stained with AdipoRed. The cells were observed under phase contrast (PC) or fluorescence microscopy (Flu) to determine the numbers and sizes of LD. Histogram analyses showed increased small LDs $(<40 \mu \mathrm{m} 2)$ and decreased large $(>80 \mu \mathrm{m} 2)$ LDs after OB-1 treatments, compared to the control. Mean \pm s.d. for three independent experiments. ${ }^{*} \mathrm{P}<0.01$ by two-way ANOVA. Bar $=30 \mu \mathrm{m}$.

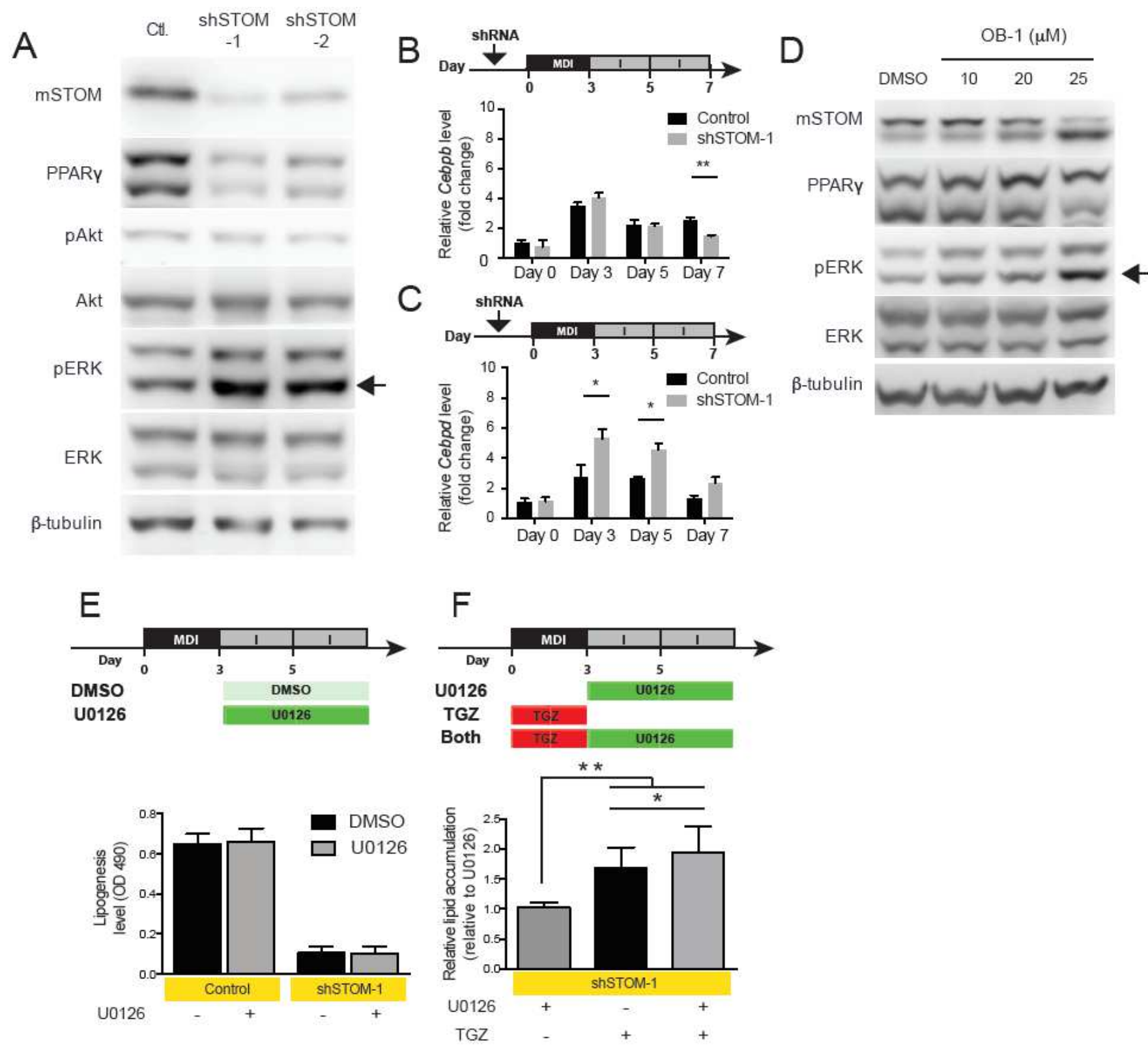

Figure 7

Knockdown of stomatin activated ERK-pathway. A) shRNA were transducted into 3T3-L1 cells to knockdown stomatin and generating shSTOM-1 and shSTOM-2 cells. After induction of differentiation, Western blotting analyses were done. Although Akt pathway remained unchanged, the ERK pathway was activated as evidenced by increased pERK (arrow). B, C) Expressions of early adipogenic genes, Cebpb and Cebpd, were examined at mRNA level by qPCR. B) Expressions of Cebpb increased in the first three days of adipogenic differentiation, and subsequently decreased. Knockdown of stomatin (exemplified by 
shSTOM-1 cells) did not significantly influence this pattern. C) Expressions of Cebpd gene also exhibited a transient early increase then declined; however, knockdown of stomatin appeared to significantly increase and maintain Cebpd expression at a relatively higher level than the control. The house-keeping Nono gene was used as the reference for qPCR exoeriments. ${ }^{*} \mathrm{P}<0.05$ by unpaired $\mathrm{t}$-test. $\mathrm{D}$ ) Treating adipocyte-like cells with stomatin inhibitor OB-1 also resulted in activation of ERK pathway, evidenced by increased pERK (arrow). E) Levels of lipogenesis by adipocyte-like 3T3-L1 cells were quantified by measuring OD 490 after Oil Red 0 staining. Treating shSTOM-1 cells with 10 1 M U0126, an ERK pathway inhibitor, from day3 to day 7, did not reverse the lipogenesis inhibition caused by stomatin knockdown. F) On the other hand, treating shSTOM-1 cells with TGZ, a PPARY activator, in the first three days of adipogenic differentiation, was able to partially recover the knockdown-caused lipogenesis deficit; treating shSTOM-1cells with both TGZ and U0126 was noted to further increase lipid accumulation. Mean \pm s.d. for six independent experiments. ${ }^{*} P<0.05$, and ${ }^{*}{ }^{*} \mathrm{P}<0.01$ by one-way ANOVA. $\mathrm{PPCR}$ : quantitative real-time PCR. 


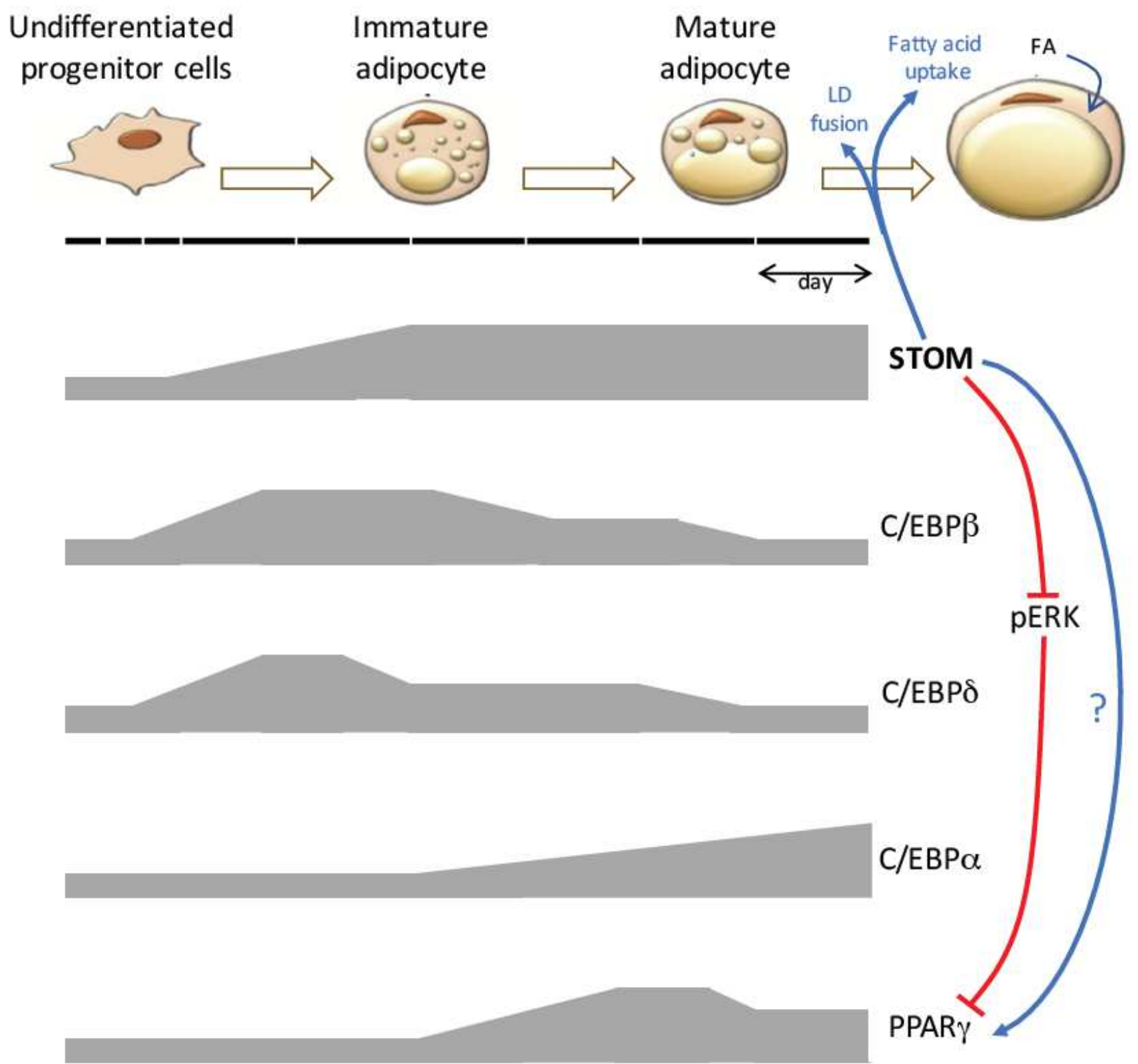

\section{Figure 8}

Stomatin's roles in modulating adipogenic differentiation and lipogenesis. Undifferentiated progenitor cells can be induced to undergo adipogenic differentiation to become immature adipocytes, and further develop into mature adipocytes, characterized by fatty acid uptake, production of lipid, and fusions and enlargements of lipid droplets (LDs). Dynamic expressions of adipogenic genes along this process are depicted. Stomatin progressively increases during adipocyte differentiation and maturation, and participates not only in fatty acid uptake and LD fusions, but also in modulation of adipogenic gene expressions. By inhibiting pERK, stomatin activates PPARY: resulting in adipocyte maturation and lipogenesis. Stomatin also plays a role in regulating early phase adipogenic genes, such as C/EBP $\beta$ and 
C/EBPS through currently unknown mechanisms. C/EBP: CCAAT/enhancer binding protein; PPARY: peroxisome proliferator-activated receptor-y.

\section{Supplementary Files}

This is a list of supplementary files associated with this preprint. Click to download.

- Supplementalvedio1LDLDfusionmovie.mp4 